ATLAS Tracker Upgrade: Silicon Strip Detectors for the SLHC

Liv Wiik on behalf of the ATLAS Collaboration

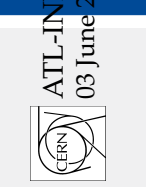

总- 


\section{The Superluminous-LHC}

\begin{tabular}{|l|l|l|l|}
\hline & LHC & SLHC Phase-1 & SLHC Phase-2 \\
\hline Peak luminosity & $10^{34} \mathrm{~cm}^{-2} \mathrm{~s}^{-1}$ & $2-3 \times 10^{34} \mathrm{~cm}^{-2} \mathrm{~s}^{-1}$ & $10^{35} \mathrm{~cm}^{-2} \mathrm{~s}^{-1}$ \\
\hline Integrated lumin & $\approx 50 \mathrm{fb}^{-1} / \mathrm{y}$ & $\approx 50 \mathrm{fb}^{-1} / \mathrm{y}$ & $\approx 500 \mathrm{fb}^{-1} / \mathrm{y}$ \\
\hline Start-up & 2009 & $\approx 2013$ & $\approx 2020$ \\
\hline
\end{tabular}

Upgrade of LHC injector chain and interaction regions

Phase-1 : Linac2 $(50 \mathrm{MeV}) \rightarrow$ Linac4 $(160 \mathrm{MeV})$ civil engineering started

Phase-2 : Booster (1.4 GeV) $\rightarrow$ LPSPL (4 GeV) PS $(25 \mathrm{GeV}) \rightarrow$ PS2 $(50 \mathrm{GeV})$

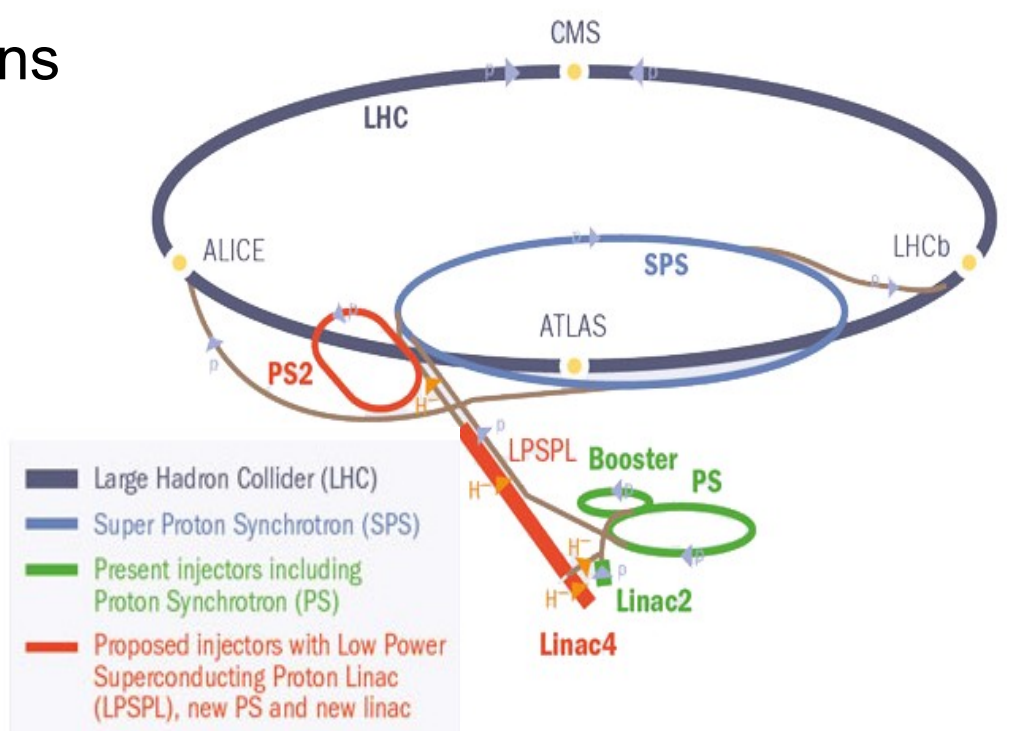




\section{The ATLAS Inner Detector at LHC}

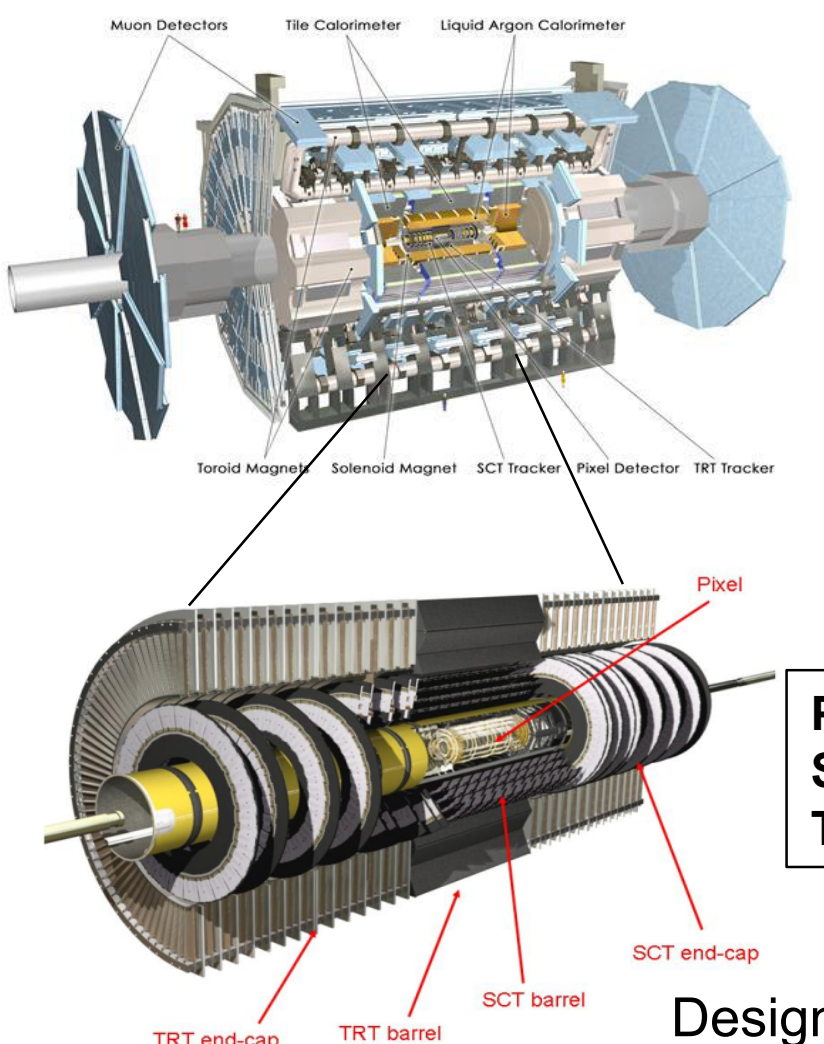

TRT end-cap TRT barrel

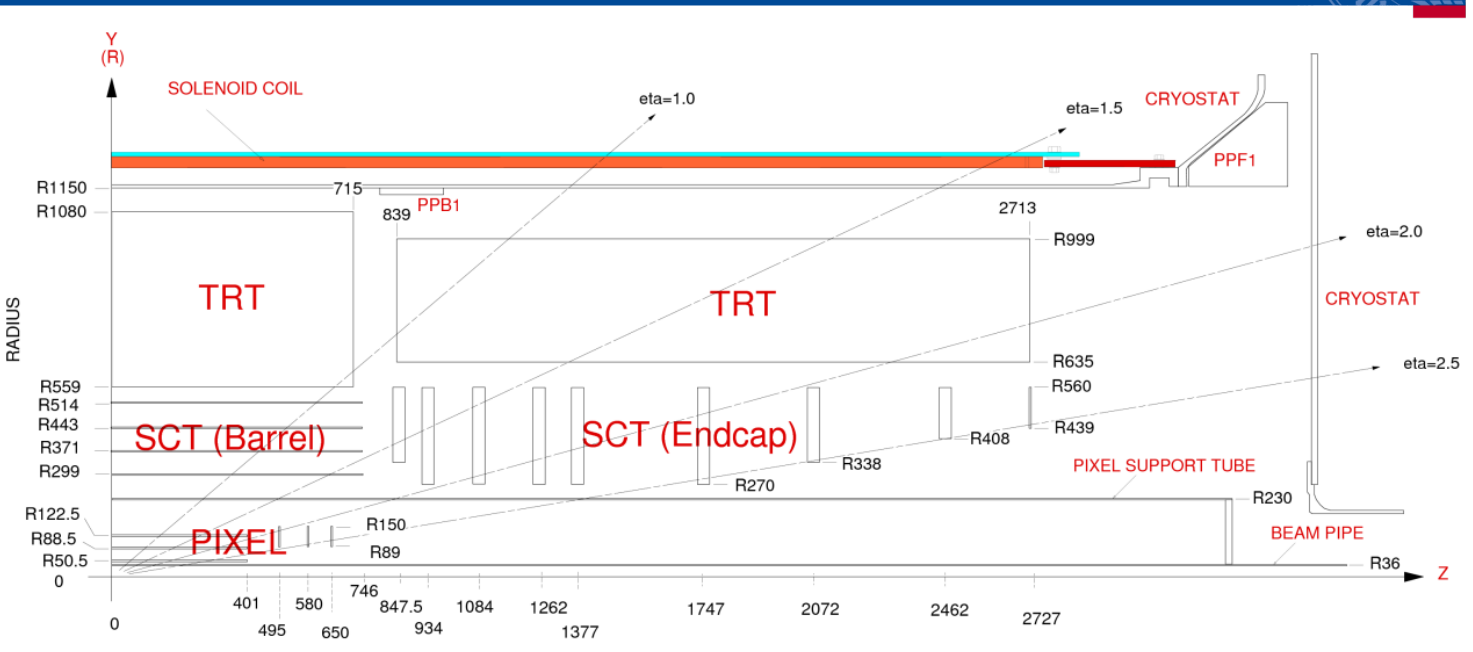

Pixel ( $n+-o n-n$ sensor technology): 3 barrels $+2 \times 3$ discs $(5<R<15 \mathrm{~cm})$

Strip (SCT) (p+-on-n sensor technology ): 4 layers $+2 \times 9$ discs $(30<R<51 \mathrm{~cm})$

TRT (4mm diameter straw drift tubes): Barrel + Wheel $(55<\mathrm{R}<105 \mathrm{~cm})$

Designed for fluences of :

- Pixel layer 0: $1 \times 10^{15} 1 \mathrm{MeV}_{\mathrm{eq}} / \mathrm{cm}^{2}$

- SCT layer 1: $2 \times 10^{14} 1 \mathrm{MeV} \mathrm{n}_{\mathrm{eq}} / \mathrm{cm}^{2}$

- TRT outer radius: $3 \times 10^{13} 1 \mathrm{MeV} \mathrm{n}_{\mathrm{eq}} / \mathrm{cm}^{2}$ 


\section{Radiation Levels at the SLHC}

\section{Particle Fluence :}

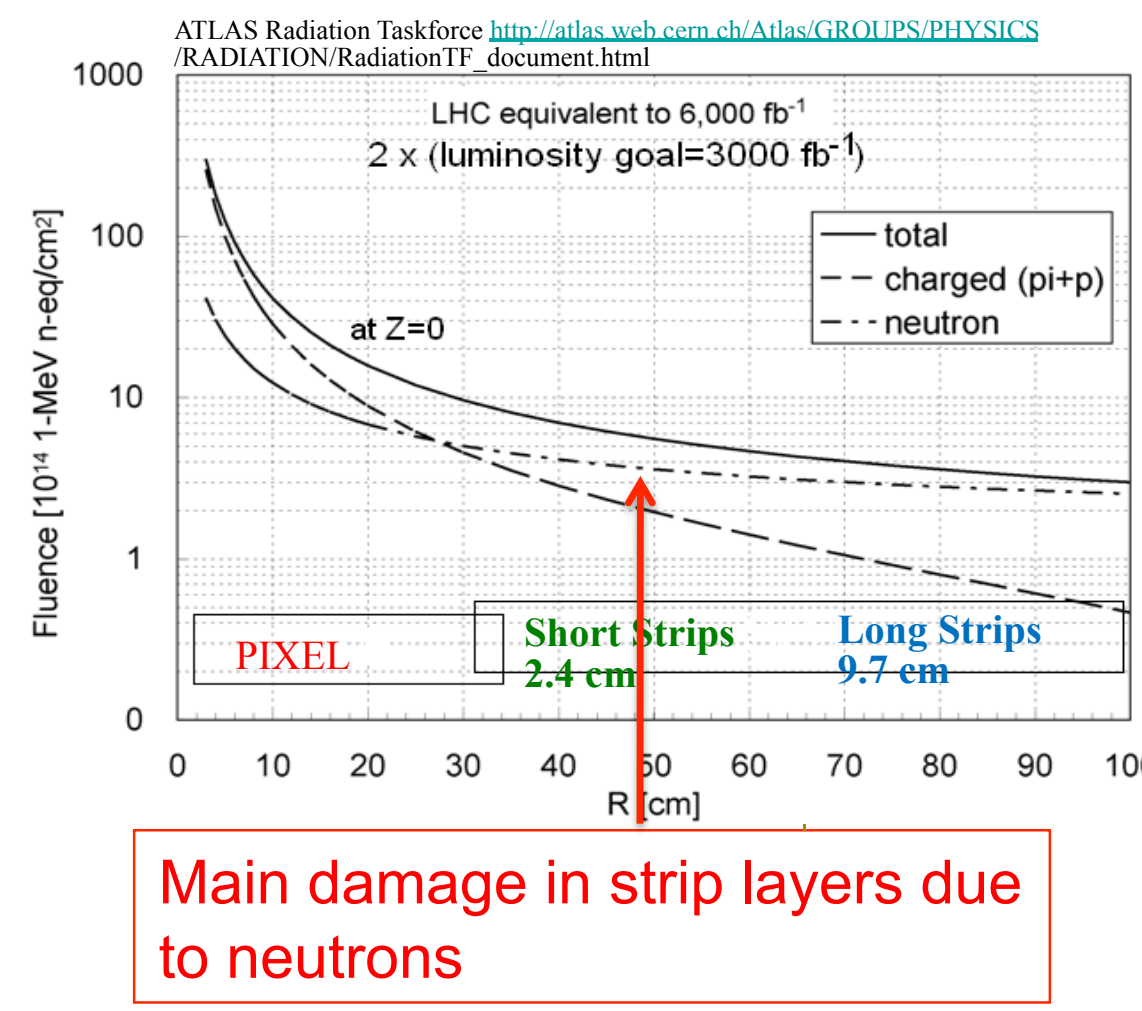

Main damage in strip layers due to neutrons

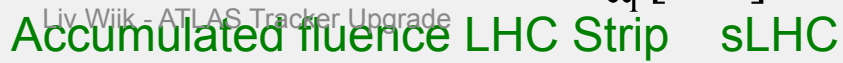

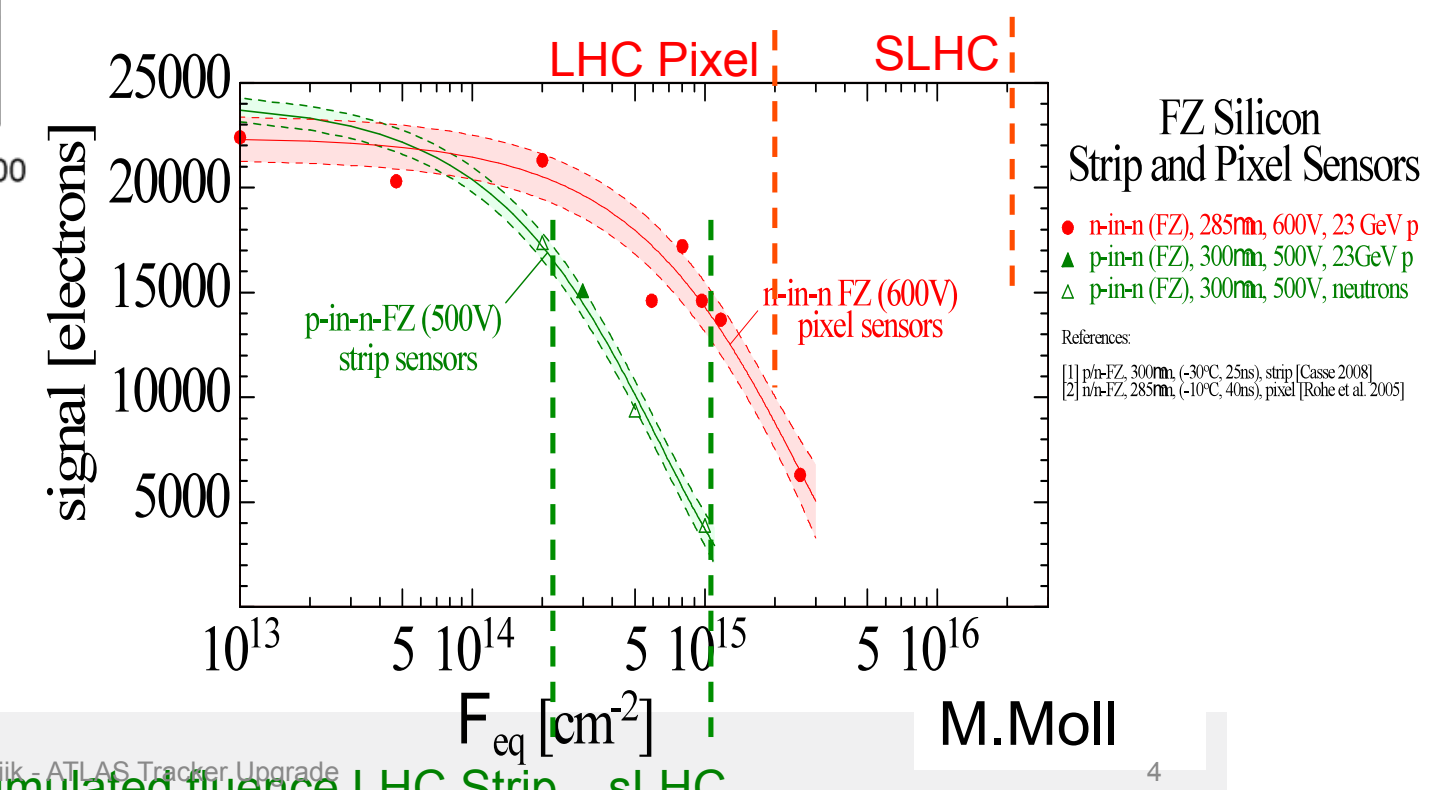

\section{Expected fluences for sensors:}

- B-layer at $3.7 \mathrm{~cm}: 2.2 \times 10^{16} 1 \mathrm{Mev} \mathrm{n} / \mathrm{cm}{ }^{2}$

- Short Strip layers: $1 \times 10^{15} 1 \mathrm{Mev} \mathrm{n}^{\mathrm{eq}} / \mathrm{cm}^{2}$

- Long Strip layer at $38 \mathrm{~cm}: 5 \times 10^{15} 1 \mathrm{Mev} \mathrm{n}^{\mathrm{eq}} / \mathrm{cm}^{2}$ 


\section{LHC vs. SLHC}

- To fully exploit SLHC physics potential existing performance of detector must be preserved if not improved

- Pile up increases from 20 events to $\sim 300-400$ events per bunch crossing

$\rightarrow$ Up to 20000 particles expected in tracker

$\rightarrow$ Finer granularity needed to keep occupancy at $1 \%$ level

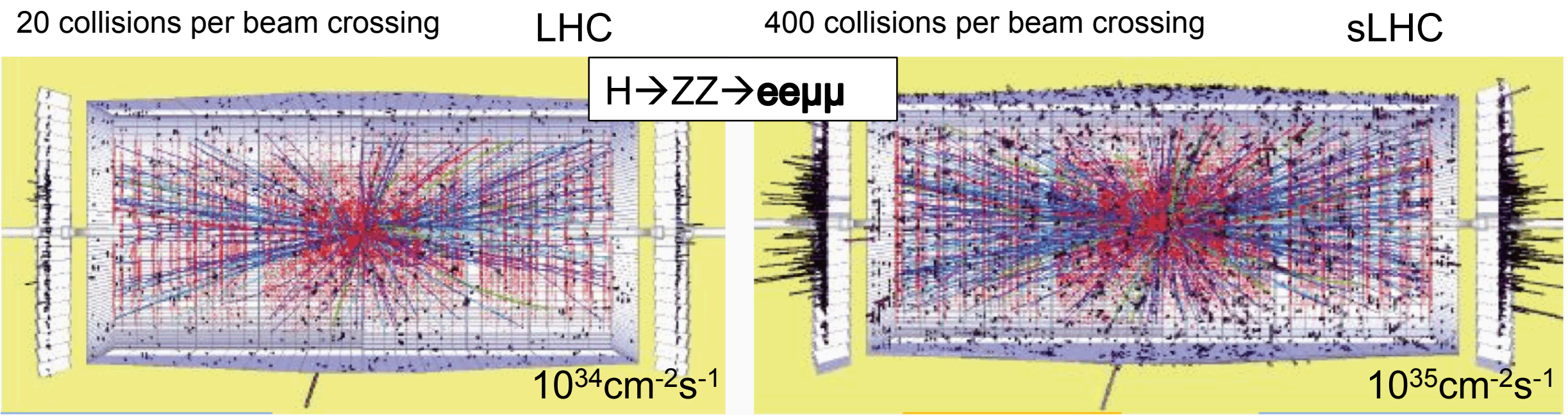

- TRT will be unable to cope with SLHC occupancy $\rightarrow$ envisage all silicon ID

- Finer granularity

$\Rightarrow \times 10$ number of channels in the same space (SCT) 


\section{Upgrades to the ATLAS Inner Detector}

- New Inner Detector layout: pixel and strip layers all silicon 亏崫

- Radiation hard technologies: n-in-p, 3D, diamond etc.

- New module integration concepts (low material budget).

- New powering schemes (serial powering or DC-DC) to reduce the number of cables.

- Improved cooling system to maintain silicon temperature below $-20^{\circ} \mathrm{C}\left(\mathrm{CO}_{2}\right.$ or $\left.\mathrm{C}_{3} \mathrm{~F}_{8}\right)$.

- Installation: limited access time inside the cavern $\rightarrow$ expect approx 14 month 


\section{Inner Detector Layout for the SLHC}

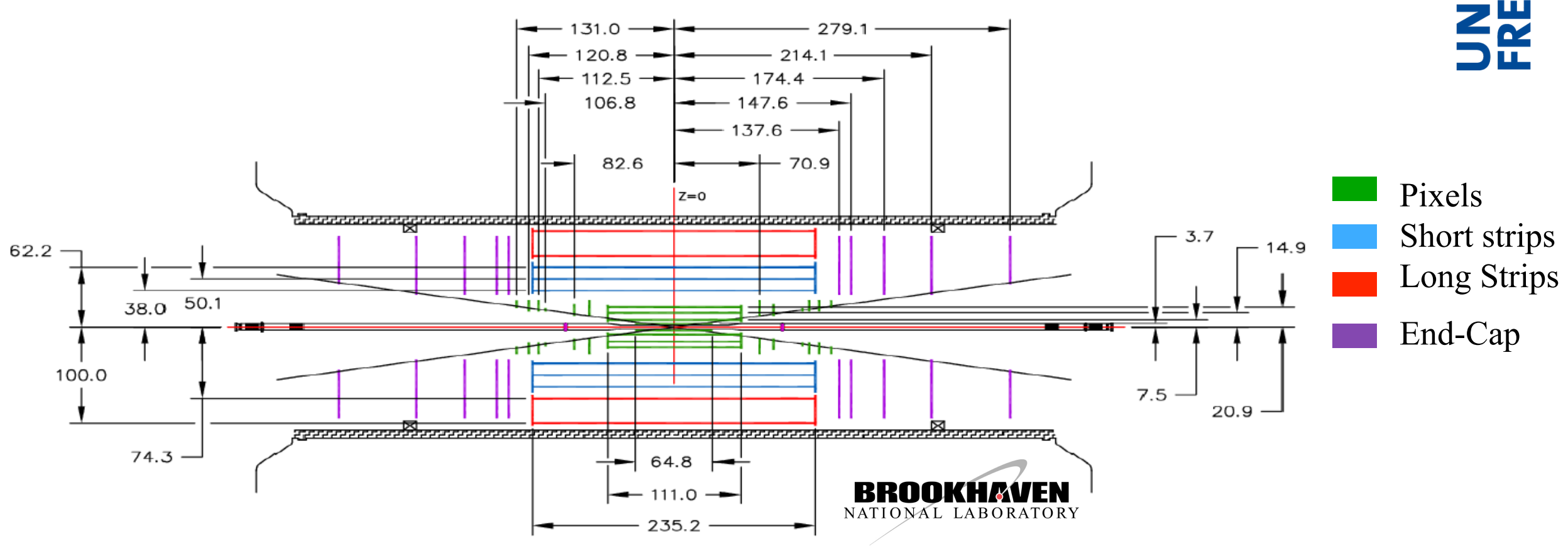

- Pixels: 4 pixel layers, 6 pixel disks $3.7-20.9 \mathrm{~cm}$

- Strips: 5 barrel layers: between 38 and $95 \mathrm{~cm}$

- 3 inner layers: SHORT STRIPS: 24 mm long

- 2 outer layers: LONG STRIPS: 96 mm long

- 5 double sided disks on each End-Cap 


\section{Radiation Hard Technologies: ATLAS07}

\section{- 6 inch (150 mm) wafers, produced by Hamamatsu Photonics K.K.}

- FZ, n-in-p

- P-stop and p-stop+p-spray isolation

- Pitch $74.5 \mu \mathrm{m}$, thickness $320 \mu \mathrm{m}$

- Miniature sensors $(1 \mathrm{~cm} \times 1 \mathrm{~cm}, 128$ strips $) \rightarrow$ For irradiation studies

- Full size prototype sensors $(9.75 \mathrm{~cm} \times 9.75 \mathrm{~cm}, 4$ rows of 1280 strips each $2.4 \mathrm{~cm}$ long $\rightarrow 5120$ strips in total)

\section{The sensors are being developed by:}

\section{ATLAS Upgrade Silicon Strip Detector Collaboration:}

H. Chen, J. Kierstead, Z. Li, D. Lynn, Brookhaven National Laboratory

J.R. Carter, L.B.A. Hommels, D. Robinson, Cavendish Laboratory, University of Cambridge,

K. Jakobs, M. Köhler, U. Parzefall, L. Wiik, Physikalisches Institut, Universität Freiburg,

A. Clark, D. Ferrèrre, S. Gonzalez Sevilla , University of Geneva

R. Bates, C. Buttar, F. Doherty, L. Eklund, V. O'Shea, University of Glasgow,

Y. Unno, S. Terada, Y. Ikegami, T. Kohriki, KEK

A. Chilingarov, H. Fox, Physics Department, Lancaster University,

A. A. Affolder, P. P. Allport, H. Brown , G. Casse, A. Greenall, M. Wormald, Department of Physics, University of Liverpool

V. Cindro, G. Kramberger, I. Mandić, M. Mikuž, Jožef Stefan University of Ljubljana,

I. Gorelov, M. Hoeferkamp, J. Metcalfe, S. Seidel, K. Toms, University of New Mexico

Z. Dolezal, P. Kodys, Charles University in Prague, Faculty of Mathematics and Physics

J.Bohm, M.Mikestikova, Academy of Sciences of the Czech Republic

C. Betancourt, N. Dawson, V. Fadeyev, M. Gerling, A. A. Grillo, S. Lindgren, P. Maddock, F. Martinez-McKinney, H. F.-W.

Sadrozinski, S. Sattari, A. Seiden, J. Von Wilpert, J. Wright

SCIPP, UC Santa Cruz

I. Dawson, R. French, L. Nicolas, S. Paganis, D. Tsionou, The University of Sheffield,

B. DeWilde, R. Maunu, D. Puldon, R. McCarthy, D. Schamberger, Stony Brook University

K. Hara, H. Hatano, S. Mitsui, M. Yamada, University of Tsukuba

M. Miñano, C. García, C. Lacasta, S. Martí i Garcia, U. Soldevila , IFIC (Centro Mixto CSIC-UVEG)

and K. Yamamura, S. Kamada, Hamamatsu Photonics K.K.

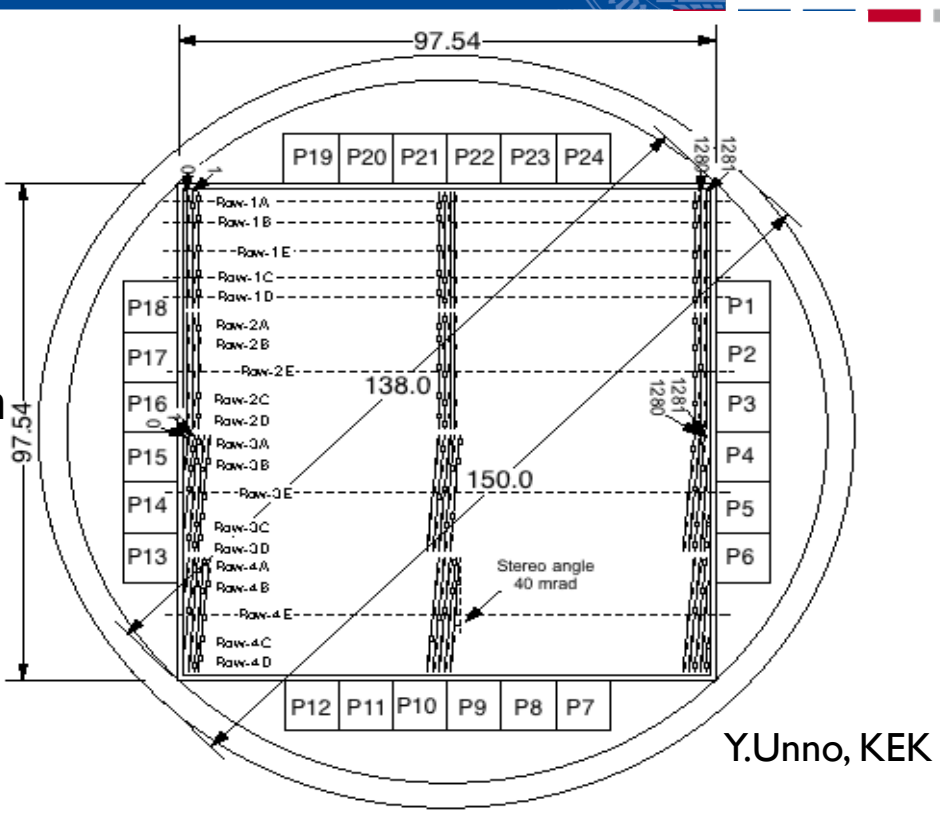

Axial-Stereo Sensor 


\section{ATLAS07 Mini Strip Sensors: CC}
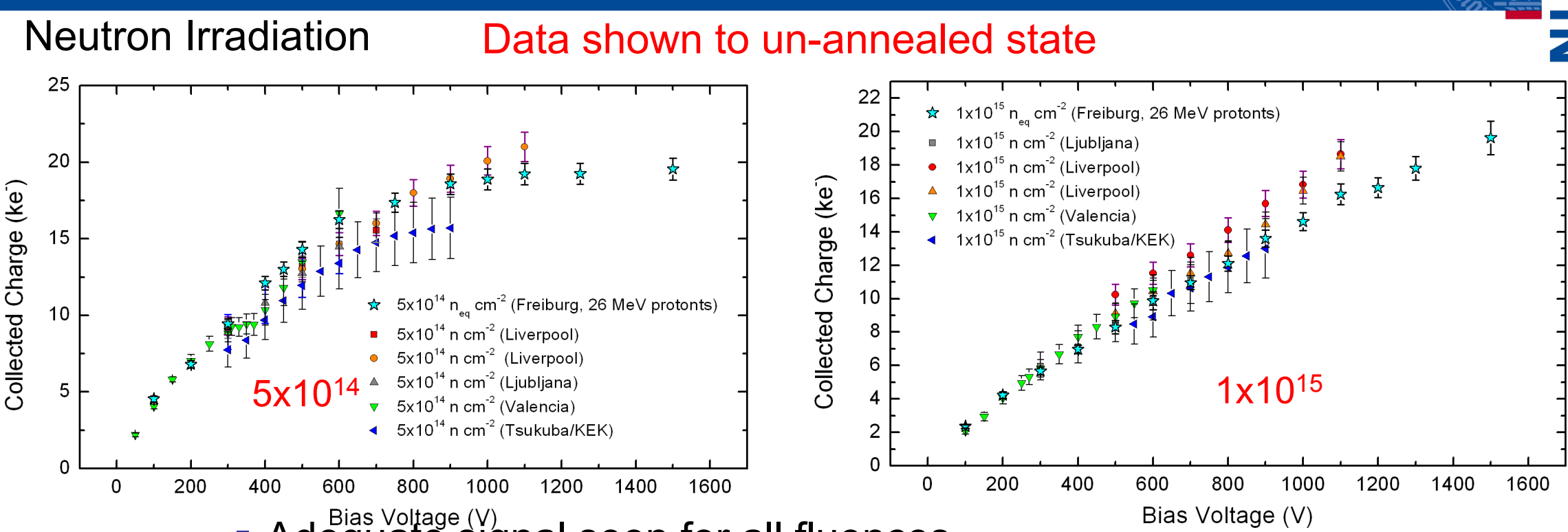

- Adequalte signal seen for all fluences

- Slight normalization/annealing correction errors visible

\begin{tabular}{|c|c|}
\hline $500 V$ & CC \\
\hline $5 \times 10^{14} \mathrm{n} / \mathrm{cm}^{2}$ & $16-19 \mathrm{Ke}^{-}$ \\
\hline $1 \times 10^{15} \mathrm{n} / \mathrm{cm}^{2}$ & $11-14 \mathrm{Ke}^{-}$ \\
\hline
\end{tabular}




\section{ATLAS07 Mini Strip Sensors: CC}
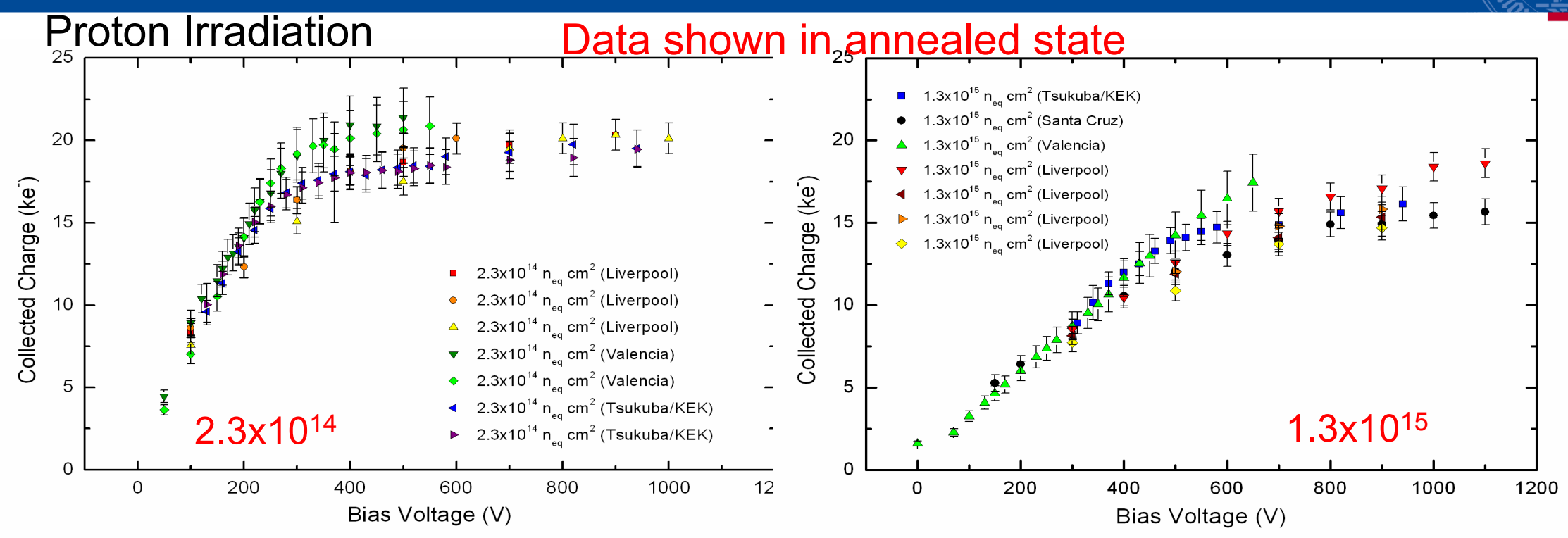

- Adequate signal seen for both fluences

- Slight normalization/annealing correction errors visible

\begin{tabular}{|c|c|}
\hline $500 \mathrm{~V}$ & $\mathbf{C C}$ \\
\hline $2.3 \times 10^{14} \mathrm{n} / \mathrm{cm}^{2}$ & $16-18 \mathrm{Ke}^{-}$ \\
\hline $1.3 \times 10^{15} \mathrm{n} / \mathrm{cm}^{2}$ & $11-14 \mathrm{Ke}^{-}$ \\
\hline
\end{tabular}




\section{ATLAS07 Mini Strip Sensors: CC @ 500 V}

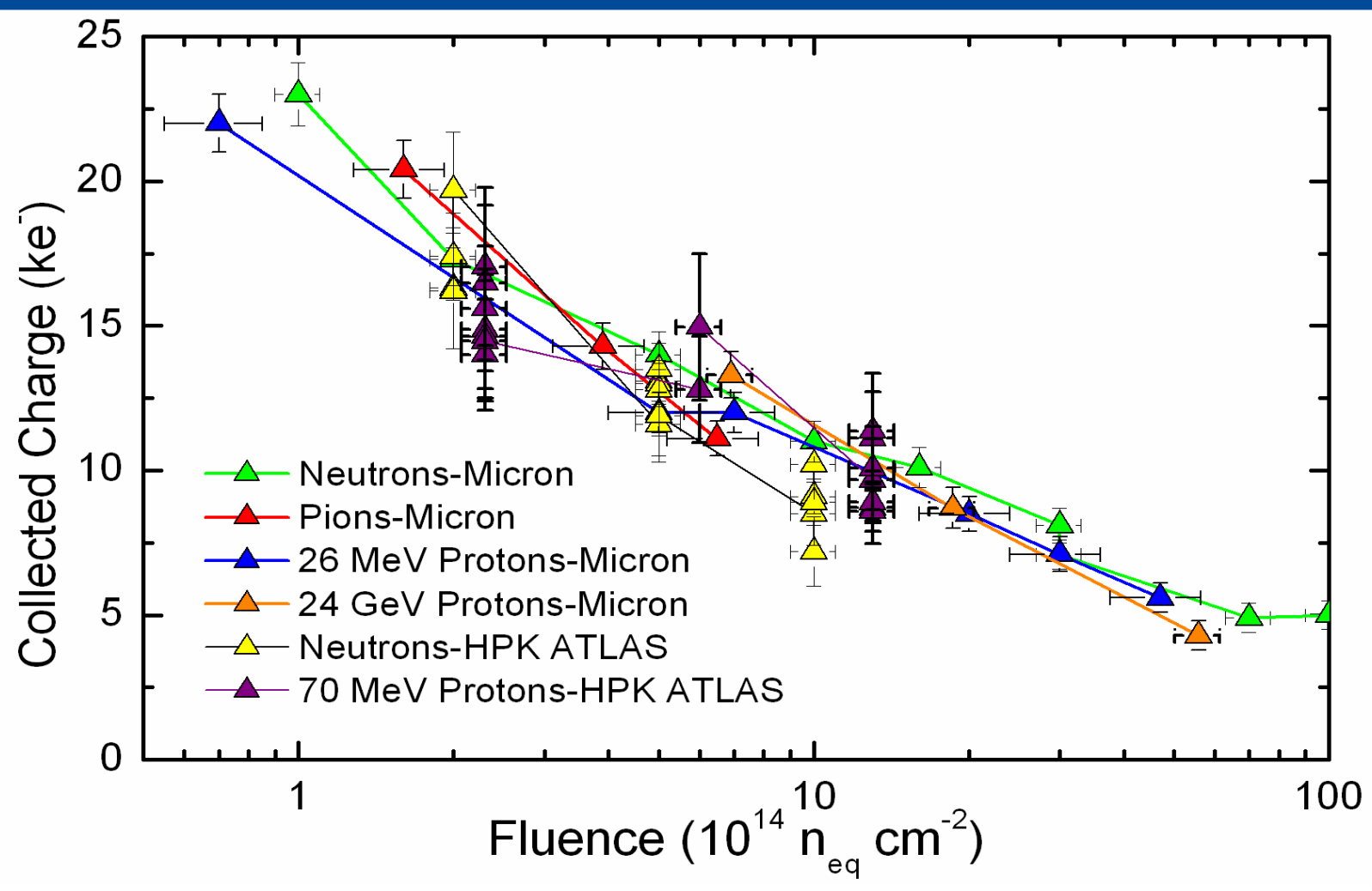

HPK data shown from all sites (with annealing corrections, i.e. CCE reduced by $-20 \%+/-10 \%)$. Pion irradiation measurements corrected for annealing during run

T.Affolder

\begin{tabular}{|c|c|c|c|c|}
\hline $500 \mathrm{~V}$ & CC & $\begin{array}{c}\text { Expected } \\
\text { noise }\end{array}$ & Strip length & $\begin{array}{c}\text { S/N } \\
\text { achievable }\end{array}$ \\
\hline $5.6 \times 10^{14} \mathrm{n} / \mathrm{cm}^{2}$ & $13 \mathrm{Ke}^{-}$ & $1186 \mathrm{e}^{-}$ & $95 \mathrm{~mm}$ & $\sim 11$ \\
\hline $1.2 \times 10^{15} \mathrm{n} / \mathrm{cm}^{2}$ & $10.6 \mathrm{Ke}^{-}$ & $607 \mathrm{e}^{-}$ & $24 \mathrm{~mm}$ & $\sim 17.5$ \\
\hline
\end{tabular}




\section{ATLAS07: Annealing CC and reverse current}
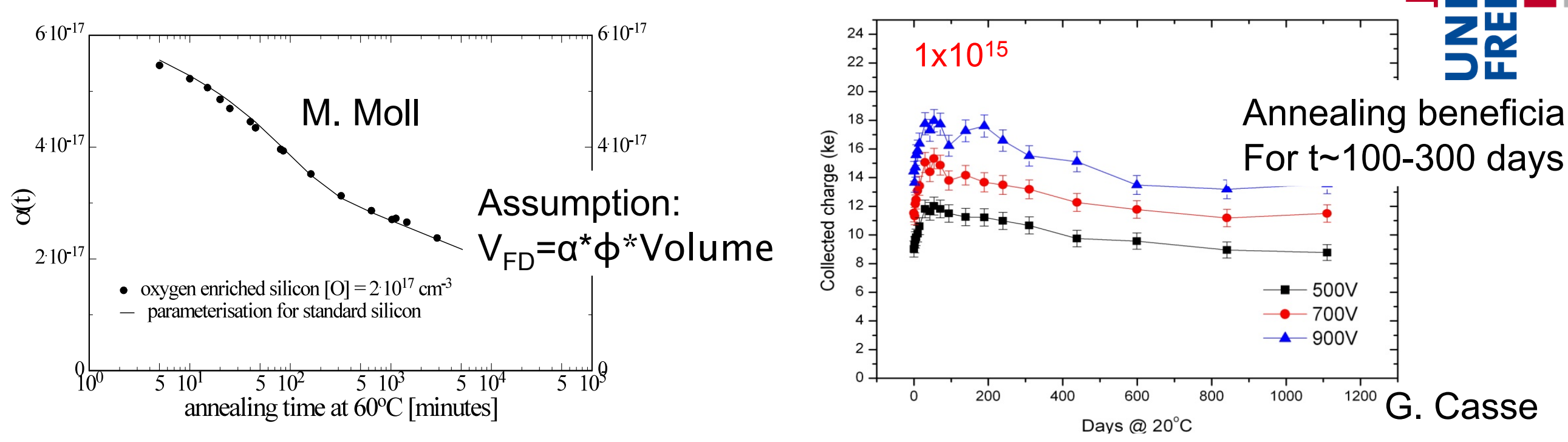

Annealing of reverse current $\rightarrow$ reduce of power dissipation

Annealing of charge collection $\rightarrow$ Recover fraction of signal in heavily irradiated silicon detectors. Even after long annealing times cc remains acceptable

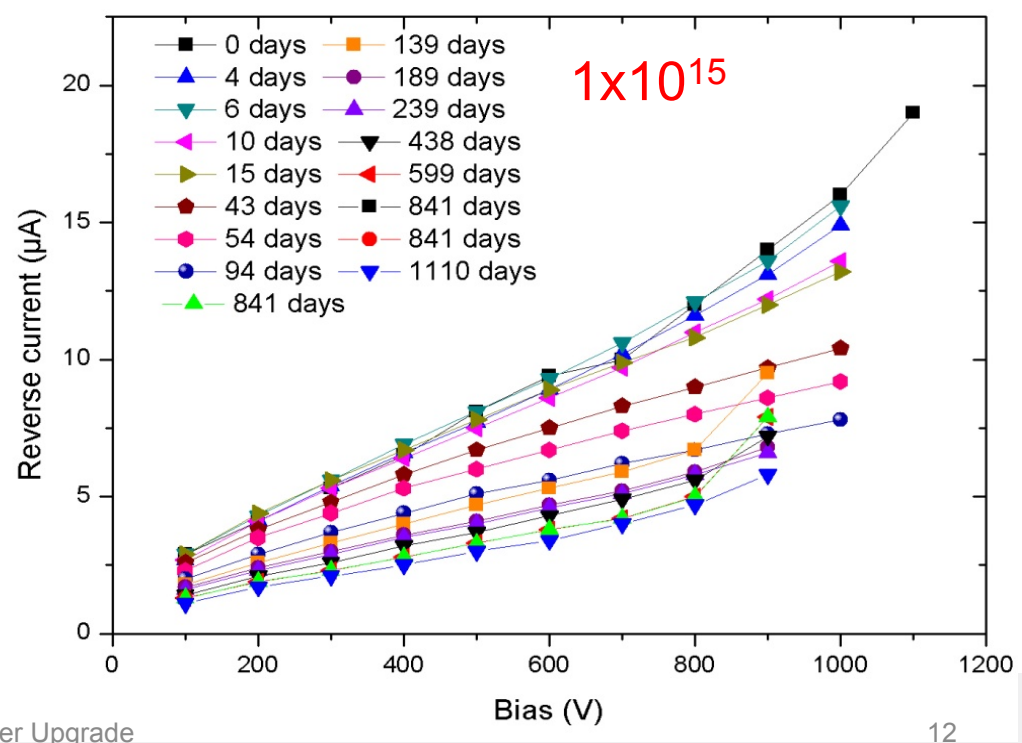




\section{Full Size Detector Testing}

\section{Total number of tested sensors: 19}

\section{ATLAS institutes involved:}

- University of Cambridge

2 sensors: W15, W16

- Stony Brook University

9 sensors: W19, W21-23, W25-29

- Institute of Physics and Charles University, Prague

- Geneva University

6 sensors: W32, W33, W35, W37, W38, W39

2 sensors: W17, W18

\section{Measurement: bias voltage scans and single strip scans}

- IV characteristics

- CV characteristics, determination of full depletion voltage

- Cint: Interstrip capacitance

- Rint: Interstrip resistance

- Ccpl: AC coupling capacitance

- Rbias: Polysilicon bias resistance

- Istrip: Leakage current to a strip

- Idiel: current through the coupling capacitance 


\section{Full Size Detector Testing}
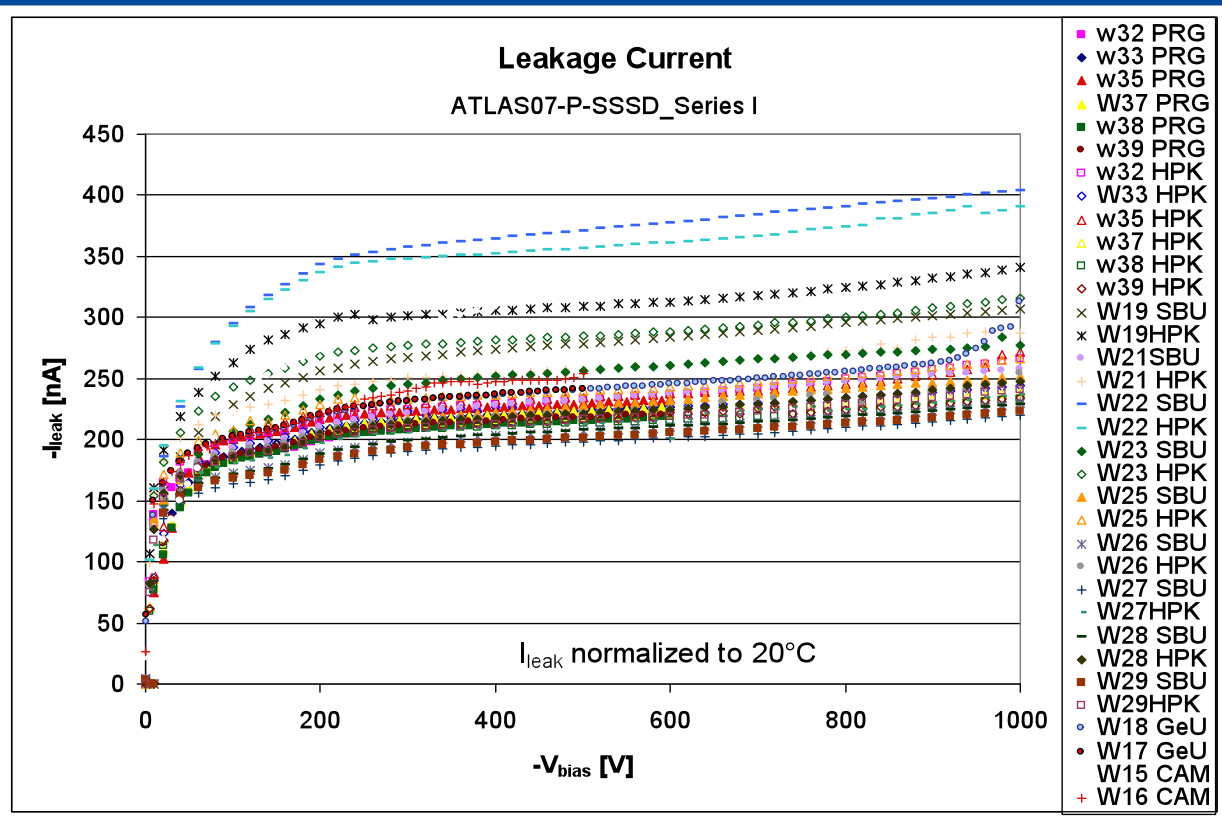

-Estimated values of Vdep:

- 6 sensors (Prague)

$199-203 \mathrm{~V}$

- 2 sensors (Cambridge) 235-245V

$\rightarrow$ All tested sensors satisfy specifications:

$$
\text { Vdep }<500 \mathrm{~V}
$$

- No microdischarges with one exception

- ATLAS07 specification:<200uA@ $600 \mathrm{~V}$ satisfied

- IV scan was repeated after bias scan and strip scan.

$\rightarrow$ Current was usually higher by $10 \%-20 \%$ and breakdown for 2 sensors at $\sim 380 \mathrm{~V}$.

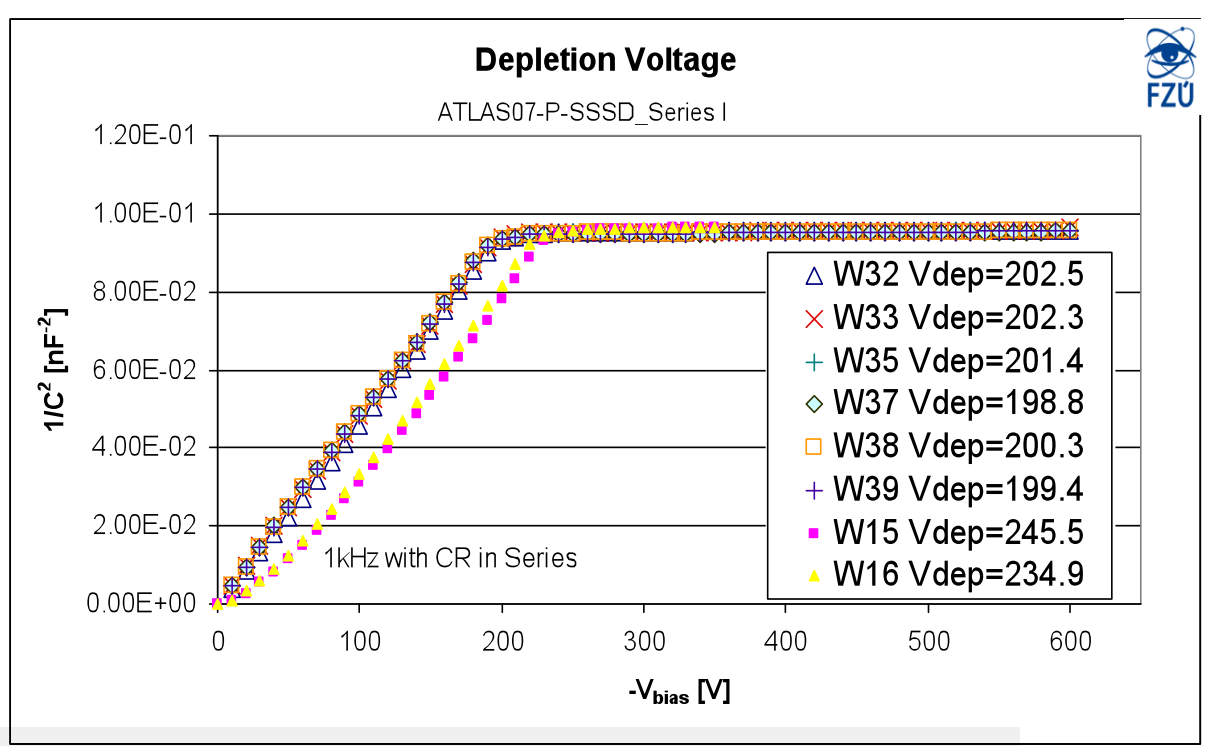




\section{Full Size Detector Testing}

\begin{tabular}{|c|c|c|}
\hline & $\begin{array}{l}\text { ATLAS07 } \\
\text { Specification }\end{array}$ & Measurement \\
\hline Leakage Current & $<200 \mu \mathrm{A}$ at $600 \mathrm{~V}$ & $\begin{array}{l}200 \mathrm{nA}- \\
370 \mathrm{nA}\end{array}$ \\
\hline Full Depletion Voltage & $<500 \mathrm{~V}$ & $\begin{array}{l}190 \mathrm{~V}- \\
245 \mathrm{~V}\end{array}$ \\
\hline $\begin{array}{l}\text { Coupling Capacitance } \\
\text { at } 1 \mathrm{kHz}\end{array}$ & $>20 \mathrm{pF} / \mathrm{cm}$ & $>28 \mathrm{pF} / \mathrm{cm}$ \\
\hline $\begin{array}{l}\text { Silicon Bias } \\
\text { Resistance }\end{array}$ & $1.5 \pm 0.5 \mathrm{M} \Omega$ & $\begin{array}{l}1.3 \mathrm{M} \Omega \\
-1.6 \mathrm{M} \Omega\end{array}$ \\
\hline $\begin{array}{l}\text { Current through } \\
\text { dielectric }\end{array}$ & $\begin{array}{l}I_{\text {diel }}<10 \mathrm{nA} \\
\text { Pinhole: } \\
>>10 \mathrm{nA}\end{array}$ & $<10 \mathrm{nA}$ \\
\hline Strip Current & No explicit limit & $<2 \mathrm{nA}$ \\
\hline $\begin{array}{l}\text { Inter-strip } \\
\text { Capacitance } \\
3 \text { probe method } \\
100 \mathrm{kHz} \\
5 \text { probe method }\end{array}$ & $<1.1 \mathrm{pF} / \mathrm{cm}$ & $\begin{array}{l}0.7 \mathrm{pF} / \mathrm{cm}- \\
0.8 \mathrm{pF} / \mathrm{cm} \\
0.66 \mathrm{pF} / \mathrm{cm}- \\
0.75 \mathrm{pF} / \mathrm{cm}\end{array}$ \\
\hline $\begin{array}{l}\text { Inter-strip Resistance } \\
\text { per cm }\end{array}$ & $\begin{array}{l}>10 \times R_{\text {bias }} \sim \\
15 \mathrm{M} \Omega\end{array}$ & $>150 \mathrm{G} \Omega$ \\
\hline
\end{tabular}

All tested sensors satisfied technical specification of non-irradiated sensors

Strip scans performed on 6 sensors showed no defects on 23040 strips: no pinholes, punch-through defects, shorts or openings of metal strips 


\section{ATLAS07 vs 3D}

3D:

- Reducing drift distance by etching $\mathrm{n}^{+}$and $\mathrm{p}^{+}$columns into silicon bulk

$\rightarrow$ Lower depletion voltage, hence less power consumption

$\rightarrow$ Less trapping, more radiation hardness

But:

- Bit lower performance of perpendicular tracks

- Challenging production
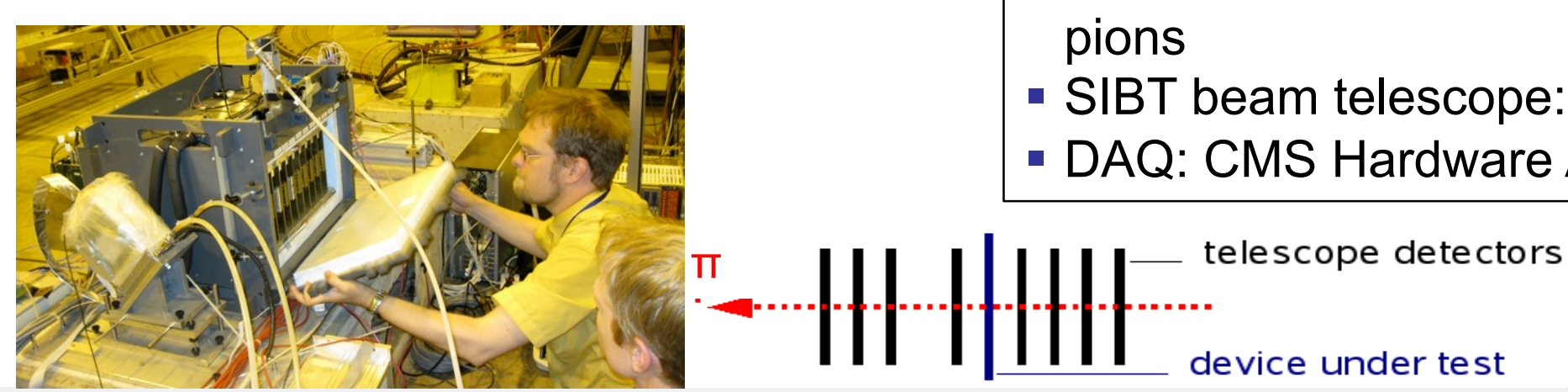

Testbeam July 2009:

- Direct comparison of 3D and ATLAS07

- Cern SPS H2 beamline using $225 \mathrm{GeV}$ pions

- SIBT beam telescope: $4 \mu \mathrm{m}$ resolution

- DAQ: CMS Hardware APV25 


\section{Planar vs 3D}
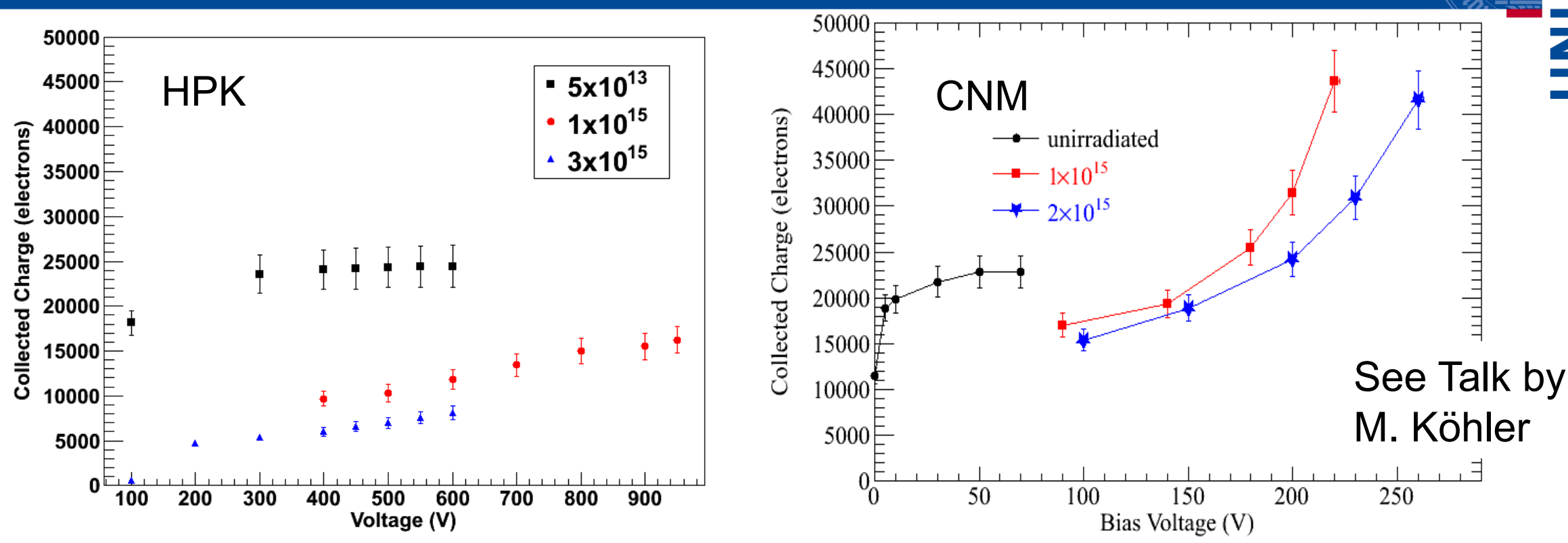

- Much lower bias voltage needed for 3D- detectors

- 3D-detectors show charge multiplication above $\sim 170 \mathrm{~V}$

$\rightarrow$ More studies needed to fullly understand this effect and possible benefits

$\rightarrow$ However, planar ATLAS07 sensors do see enough charge 


\section{Module Integration Concepts: Barrel}

Stave: Hybrid glued to sensors. These glued to bus tape, glued to cooling substrate.

$60 \mathrm{~cm}, 9 \mathrm{~cm}$ strip, 4 chips wide

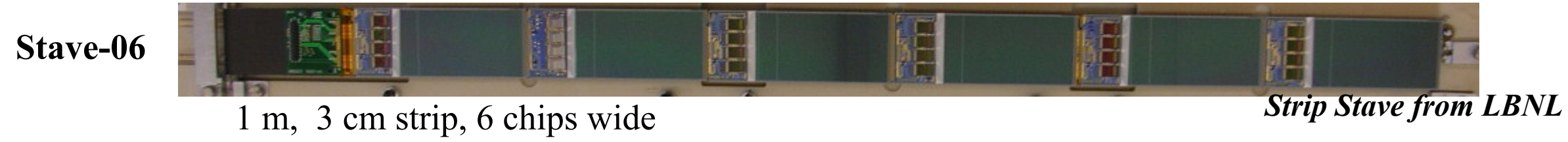

Stave-07

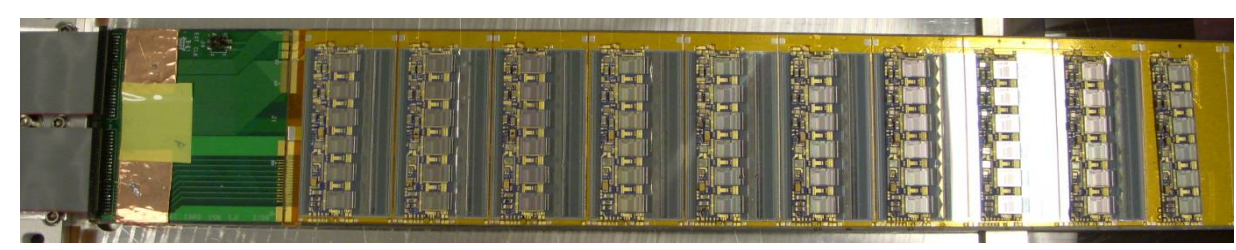

- Individual hybrids/modules work well electrically. Good noise performance.

- Tested 6 modules on stave with ABCD chips. Serial Powering lines.

- $\mathrm{CO}^{2}$ cooling foreseen

- Lots of Work ongoing Under Construction: $1.2 \mathrm{~m}, 2.5 \mathrm{~cm}$ strip, 10 chips wide (20 chips/hybrid)

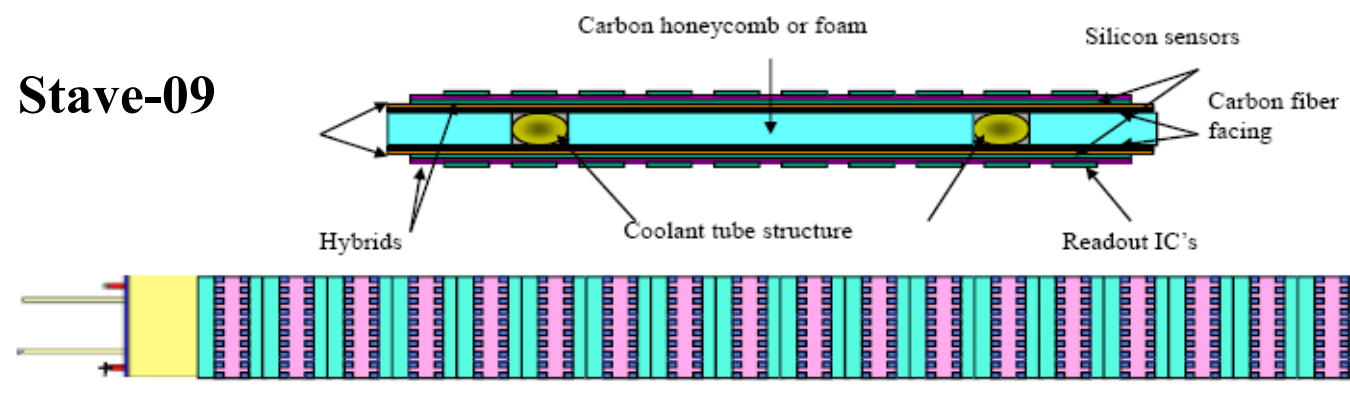

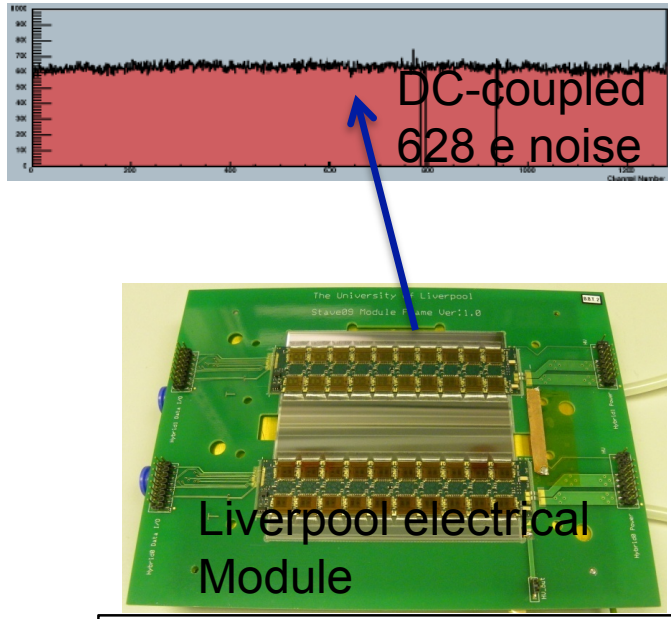

- Hybrid glued directly to active sensor side

- ABCN25 chips

- Kapton Hybrid

- Embedded Bus Cable

- Stave mechanical core

- Both hybrids are readout in parallel (40xABCN-25)

- DC referenced to each other (not AC coupled) 


\section{Module Integration Concepts: Strip End-Cap}

- 5 disks on each end-cap

- 32 petals/disk (16 on each side)

- petal surface: $830 \mathrm{~cm}^{2}$

.116 chips/petal
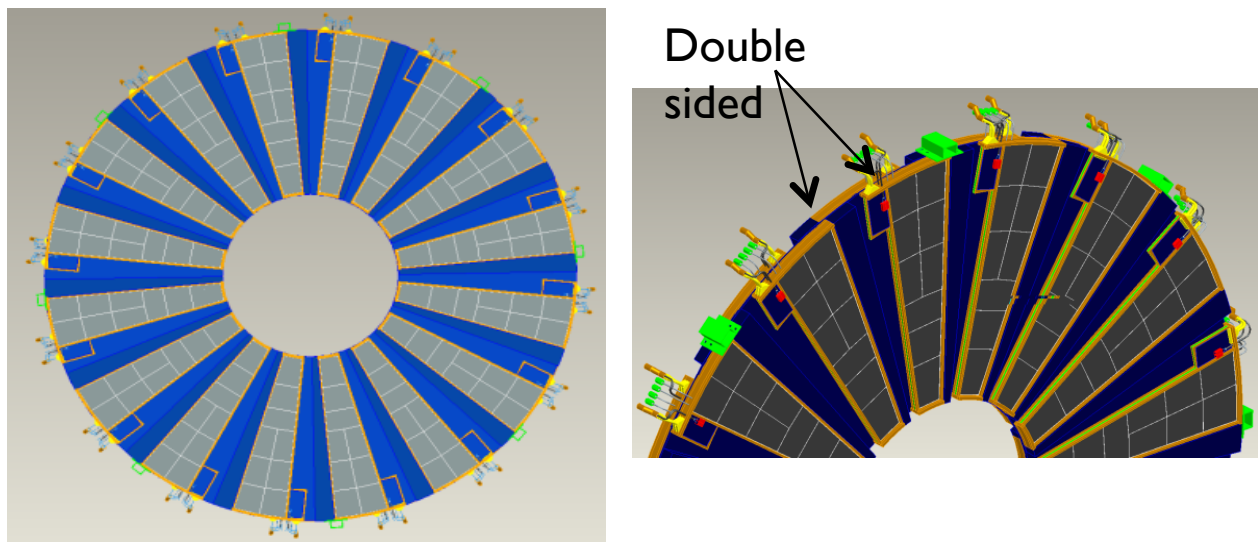

C.Lacasta (IFIC,Valencia)

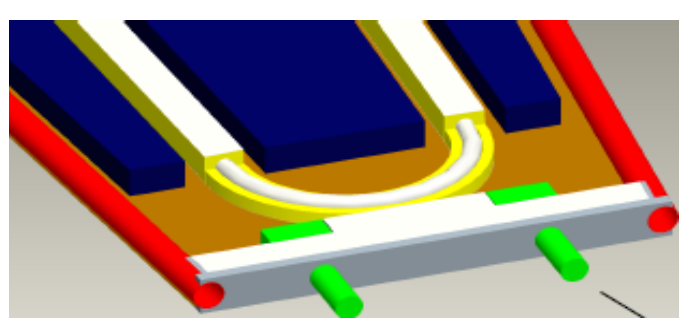

- 2 carbon facings + Honeycomb sandwich core

- Independent e-services + Bus Cable

- Independent C02 cooling pipe
- 6 Sensor rings

- 9 different hybrid types

- 6 different sensor types

- 3 short strip senors

- 2 medium strip sensors

- 1 long strip sensor 


\section{Conclusions}

- Need to replace Inner Detector for SLHC upgrade

- A lot of R\&D work has been carried out and Lol is being written

- New Inner Detecor will consist of 4 pixel layers, 5x2 pixel end-caps, 3 short strip layers (24 mm sensors) and 2 long strip layers ( $96 \mathrm{~mm}$ senors) in the barrel, $6 \times 2$ end-caps with petals

- Hamamatsu ATLAS07 production of p-type sensors, 6 inch wafers, both mini sensors and full size sensors produced

- ATLAS07 mini-strip-sensors show that technology is radiation hard enough for expected upgrade fluence

- Enough charge is collected even at high fluences

- Annealing during shut down shows positive effects on leakage current

- The full size senors satisfy by far the technical specifications

- Prototypes for module integration are being built and tested

$\rightarrow$ A large amount of work is being done, good progress 


\section{Backup}

$>$ Services:

- The required high granularity supposes (in the same space)

- x5 number of channels

- x5 number of cables

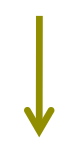

New options in powering (not individual powering)

\begin{tabular}{|c|c|c|c|c|c|}
\hline Current ID & Area $\left.\mathbf{( m}^{\mathbf{2}}\right)$ & Channels & Upgrade & Area $\left.\mathbf{( m}^{\mathbf{2}}\right)$ & Channels \\
\hline Pixels & 1.8 & $80 \mathrm{M}$ & Pixels & 5 & $\sim 300 \mathrm{M}$ \\
\hline SCT & 61 & $\sim 6.3 \mathrm{M}$ & Short Strips & 60 & $\sim 30 \mathrm{M}$ \\
\hline TRT & & $400 \mathrm{~K}$ & Long Strips & 100 & $\sim 15 \mathrm{M}$ \\
\hline
\end{tabular}




\section{Backup}

- Several options on powering: DC/DC or serial.

- Cannot have individual module powering $\rightarrow$ too much material and no space.

- Requirements: High power efficiency, low noise, safety (overcurrent, overvoltage, overtemperature).

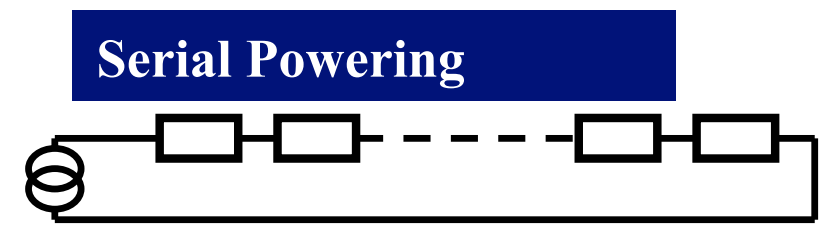

\section{DC-DC Powering}

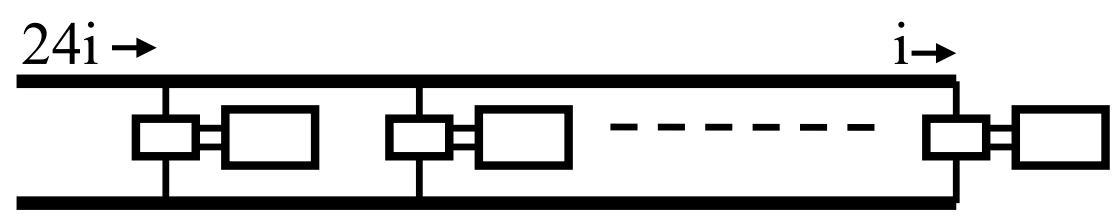

Serial Powering scheme has been shown to perform well on 6 and 30 module staves (Stave06,Stave07)

- Excellent noise performances

- Current issues:

- Protection schemes (shunt regulators) possible integration into FE chips

- Custom current source

\section{DC/DC scheme:}

- Only 1 power line/stave (10-12V)

- Distribution with 2 conversion stages:

- Stage $1 \rightarrow 2$ converters: $2.5 \mathrm{~V}$ analog and $1.8 \mathrm{~V}$ digital

- Stage $2 \rightarrow$ On-chip switched capacitor

- High granularity of the power distribution

- Very flexible 


\section{Backup: Schedule}

Somewhat outdated

\begin{tabular}{|c|c|}
\hline Milestone & Date \\
\hline Straw Man \& options fixed & Dec 2006 \\
\hline R\&D towards inner detector conceptual design & $2007-2010$ \\
\hline LoI & May 2010 \\
\hline Technical Proposal, Initial MoU and Costing & April 2012 \\
\hline Inner Tracker TDR & Dec 2013 \\
\hline Production readiness reviews and ramp up production & 2014 \\
\hline New Insertable B-layer Installation & End 2014 \\
\hline Procure parts, Component assembly & $2014-2016$ \\
\hline Surface assembly & September $2016-$ end 2017 \\
\hline Surface testing & 2018 \\
\hline Stop LHC & Sep 2018 \\
\hline Remove old detectors, install new & Oct 2018 - Dec 2019 \\
\hline Commission new detectors & Jan 2020 - Mar 2020 \\
\hline Take data & April 2020 \\
\hline
\end{tabular}




\section{Coupling Capacitance STRIP SCANS}

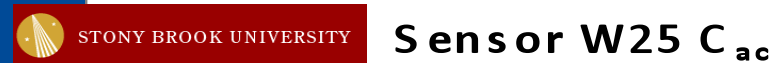

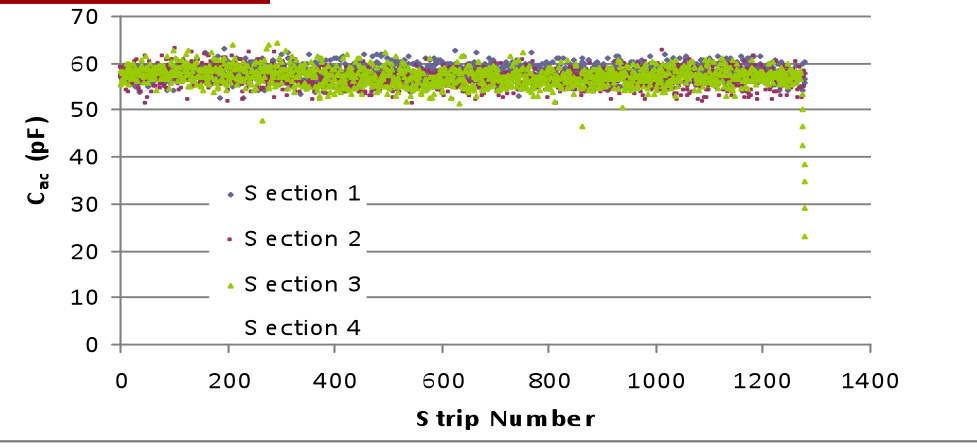

Lower capacitances expected near edges of sections 3,4

Ccpl measured between $A C$ and DC pads reached the value $66 \mathrm{pF}-68 \mathrm{pF}$ per strip.

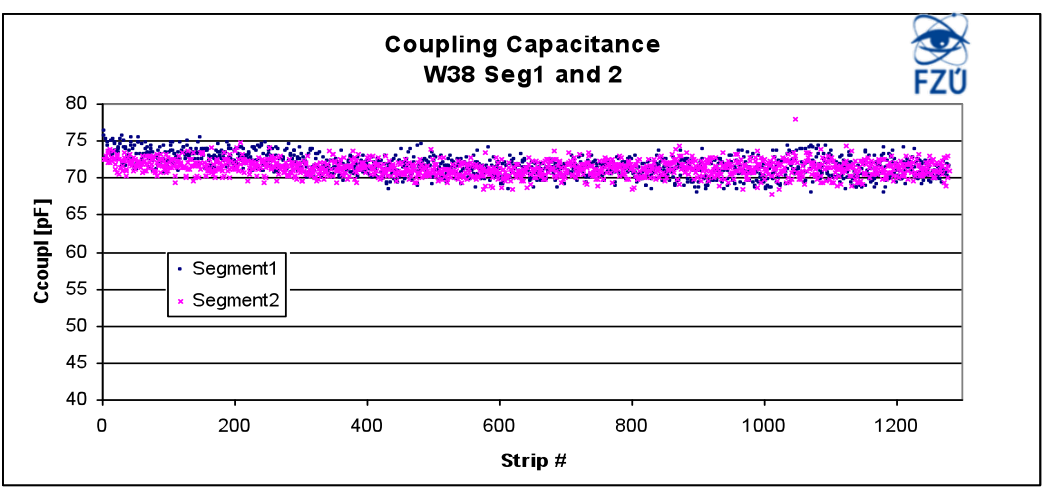

UNIVERSITYOF
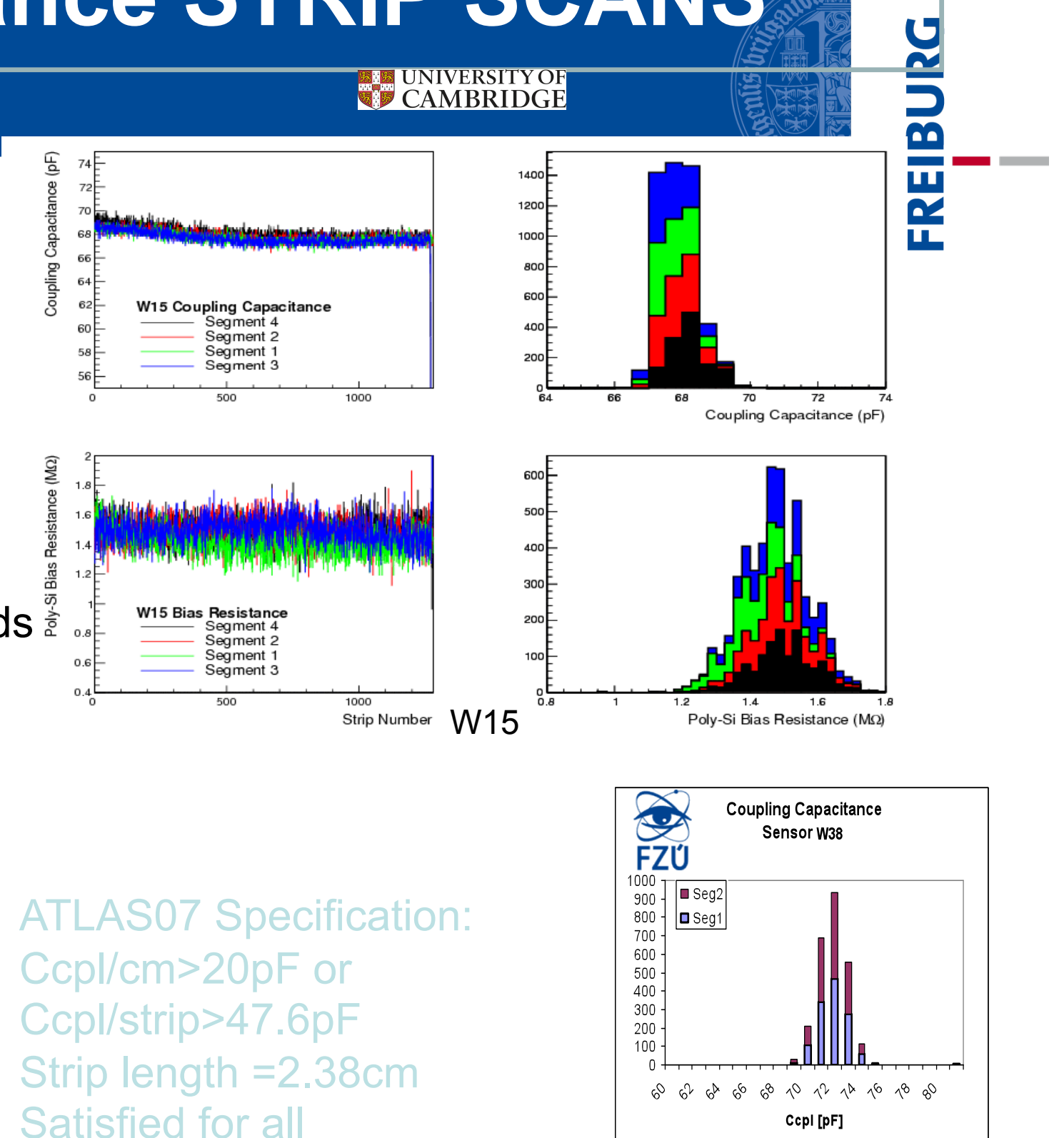

satisfied for all

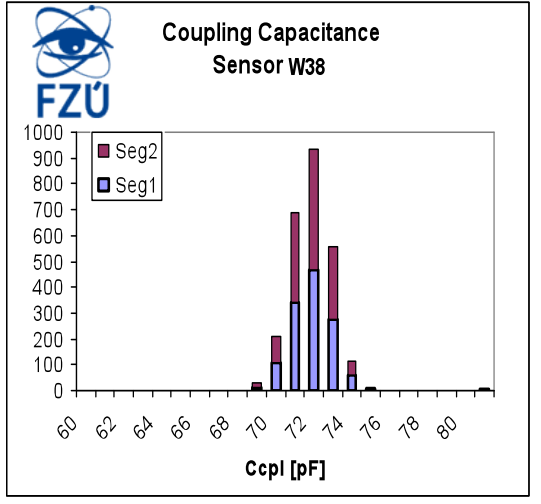




\section{Interstrip Capacitance}

\section{Measured on 6400 pairs of strips}
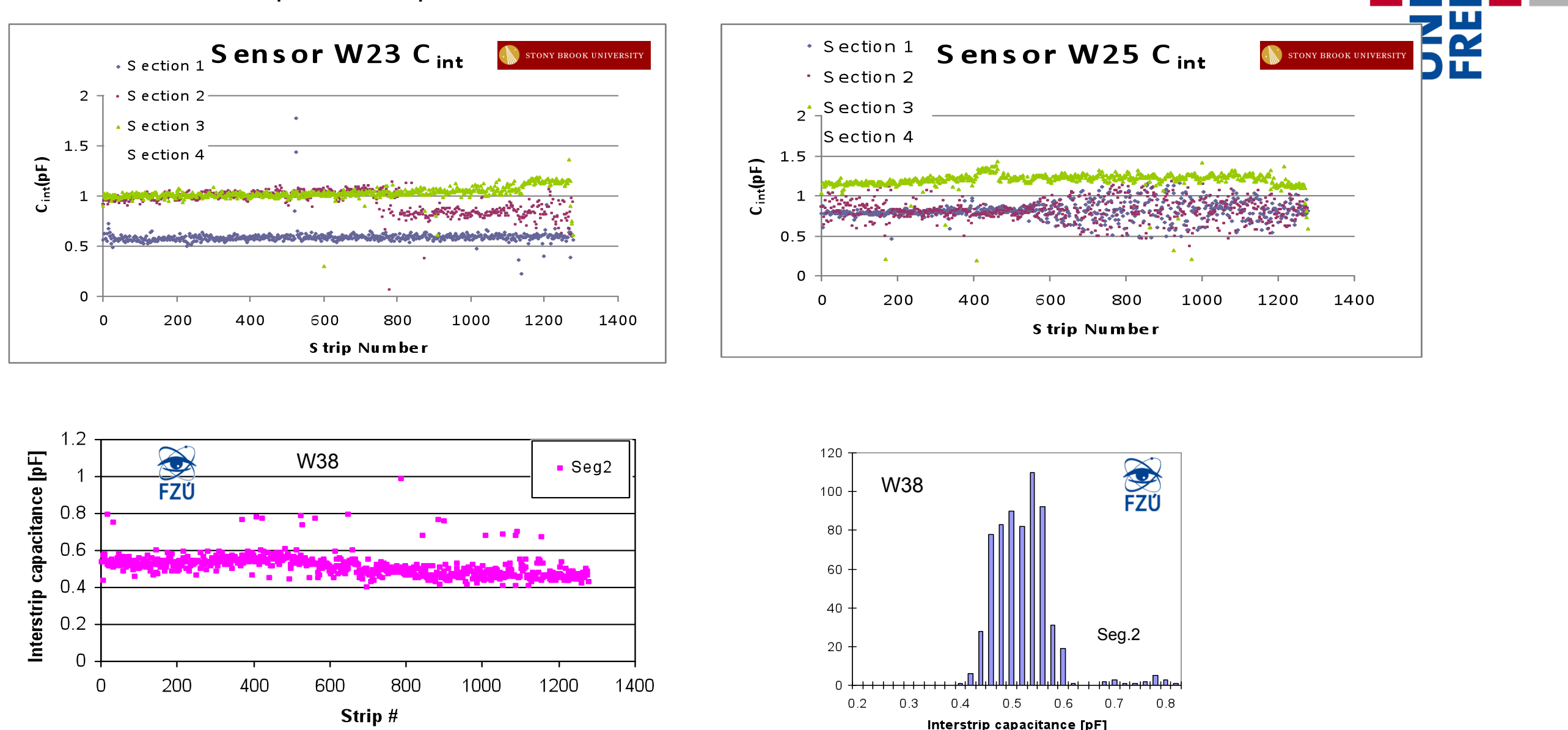

Measurements show acceptable uniformity of Cint over whole detector 


\section{Interstrip Resistance/Conductance}

Interstrip conductance: Cond $_{\text {int }}=1 /$ Rint

STONY BROOK UNIVERSITY

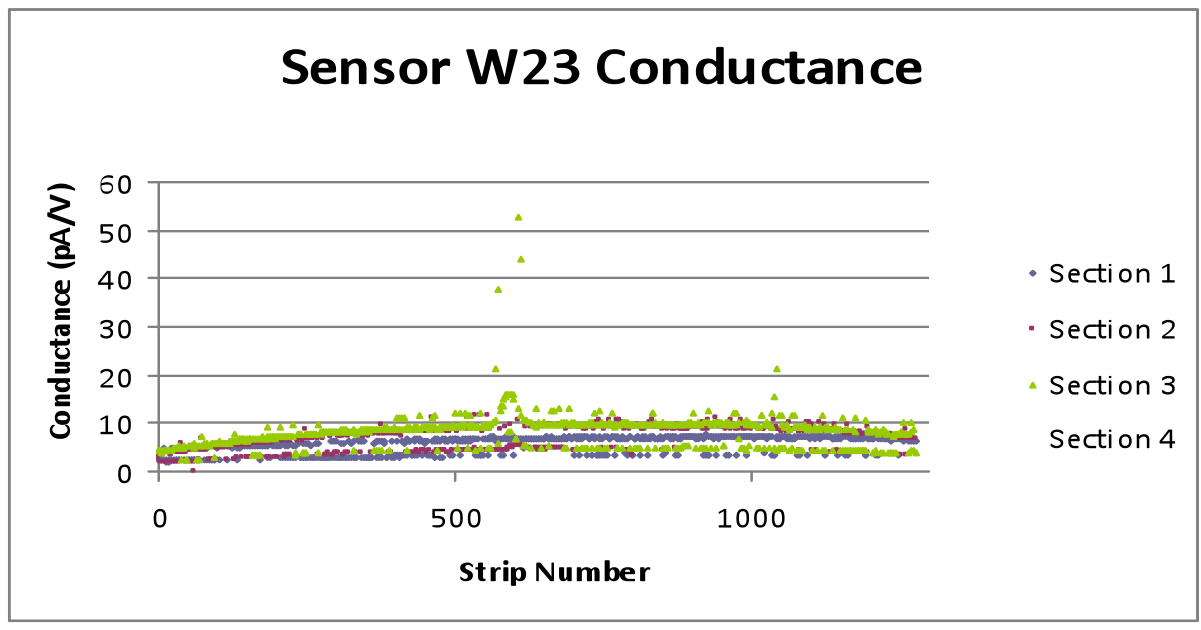

All strips have an interstrip conductance $<52 \mathrm{pA} / \mathrm{V}$ This corresponds to $\mathrm{R}_{\text {int }}>19.2 \times 10^{9} \mathrm{Ohms}$.

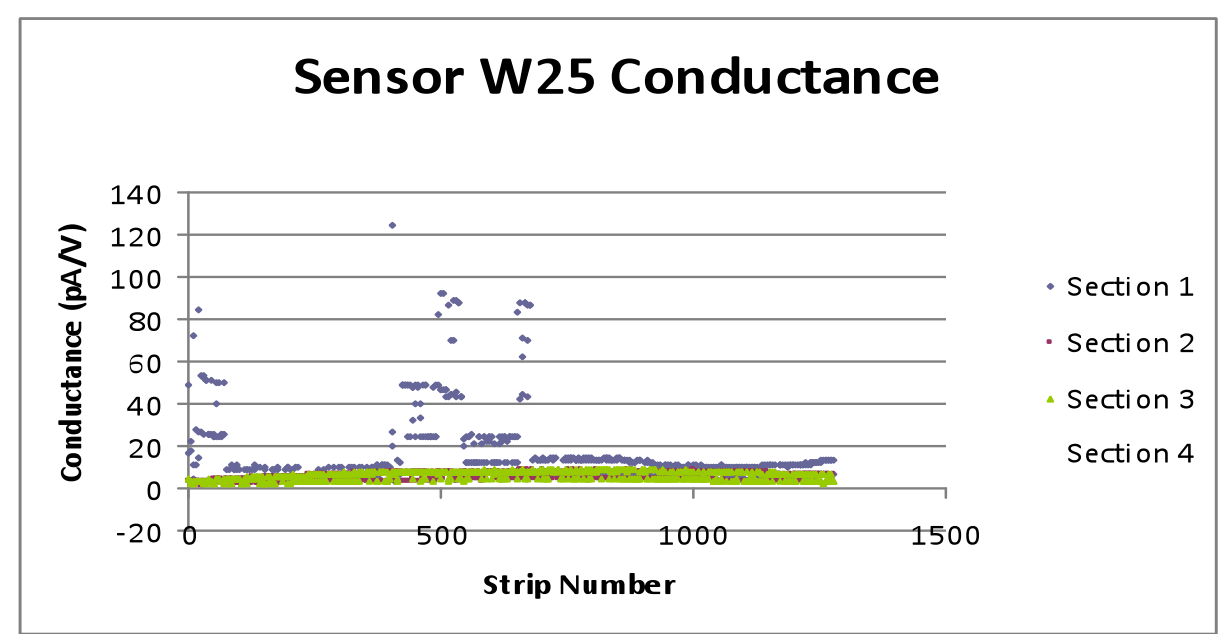

All strips have an interstrip conductance $<122 \mathrm{pA} / \mathrm{V}$. This corresponds to $\mathrm{R}_{\text {int }}>8.2 \times 10^{9}$ Ohms.

Wild variation in sections 1,4 could be a bias contact problem.

\section{Interstrip Conductance (Resistance) is uniform over whole sensor and consistent with} values measured by 3 needles.

ATLAS07 Specs: Rint>10xRbias at -200V 


\section{Backup p-type Detectors: Motivation}

The current SCT sensors use p-on-n technology. They are not sufficient radiation hard for the LHC upgrade
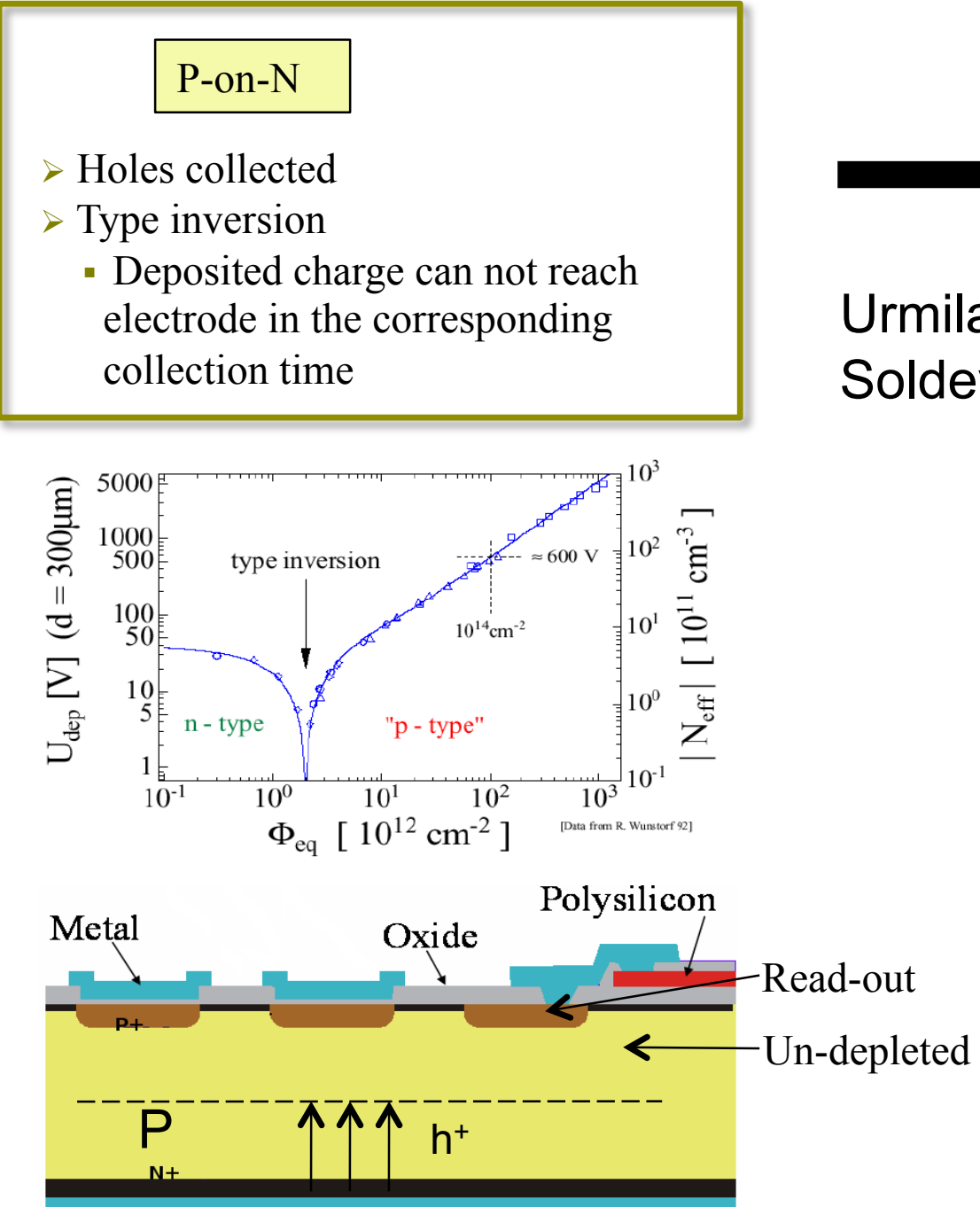

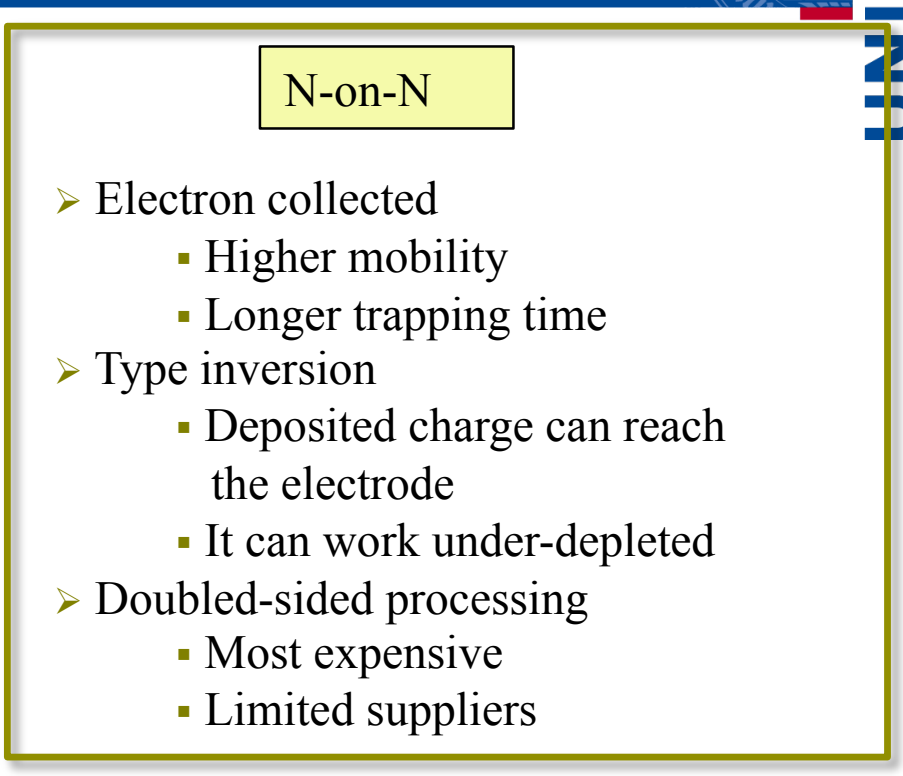

\section{Urmila Soldevilla}

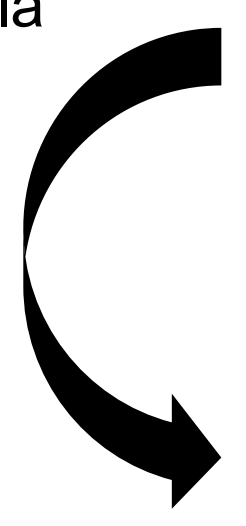




\section{Interstrip Capacitance}

\section{ATLAS07 Technical Specification: Cint@100kHz<1.1pF/cm}

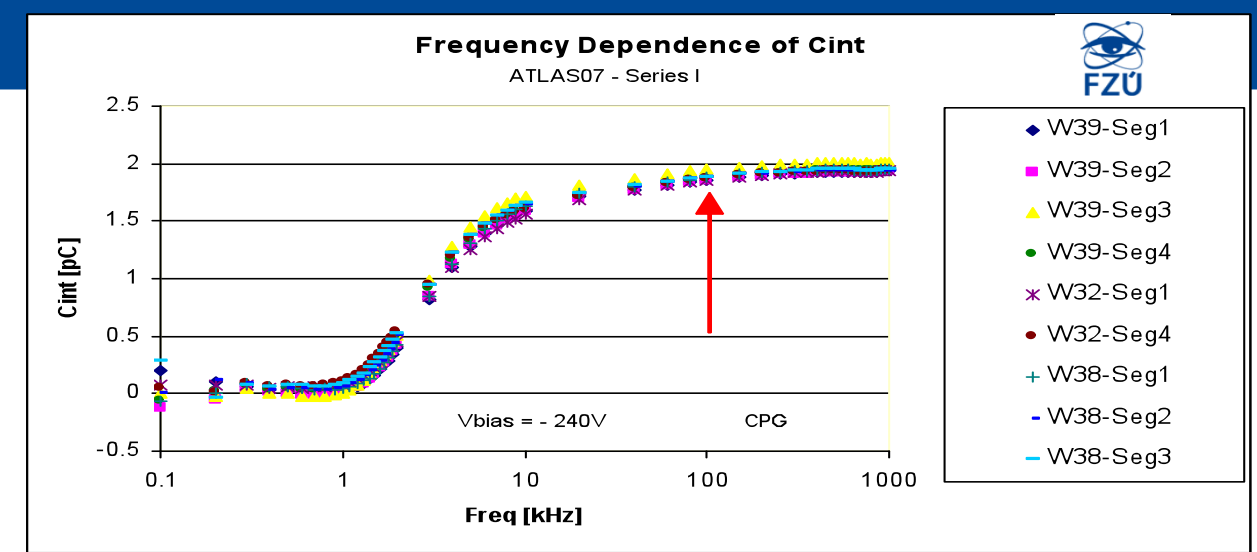

Cint is in fact saturated at $1 \mathrm{MHZ}$ but $100 \mathrm{kHz}$ is given in the ATLAS07 Specs.
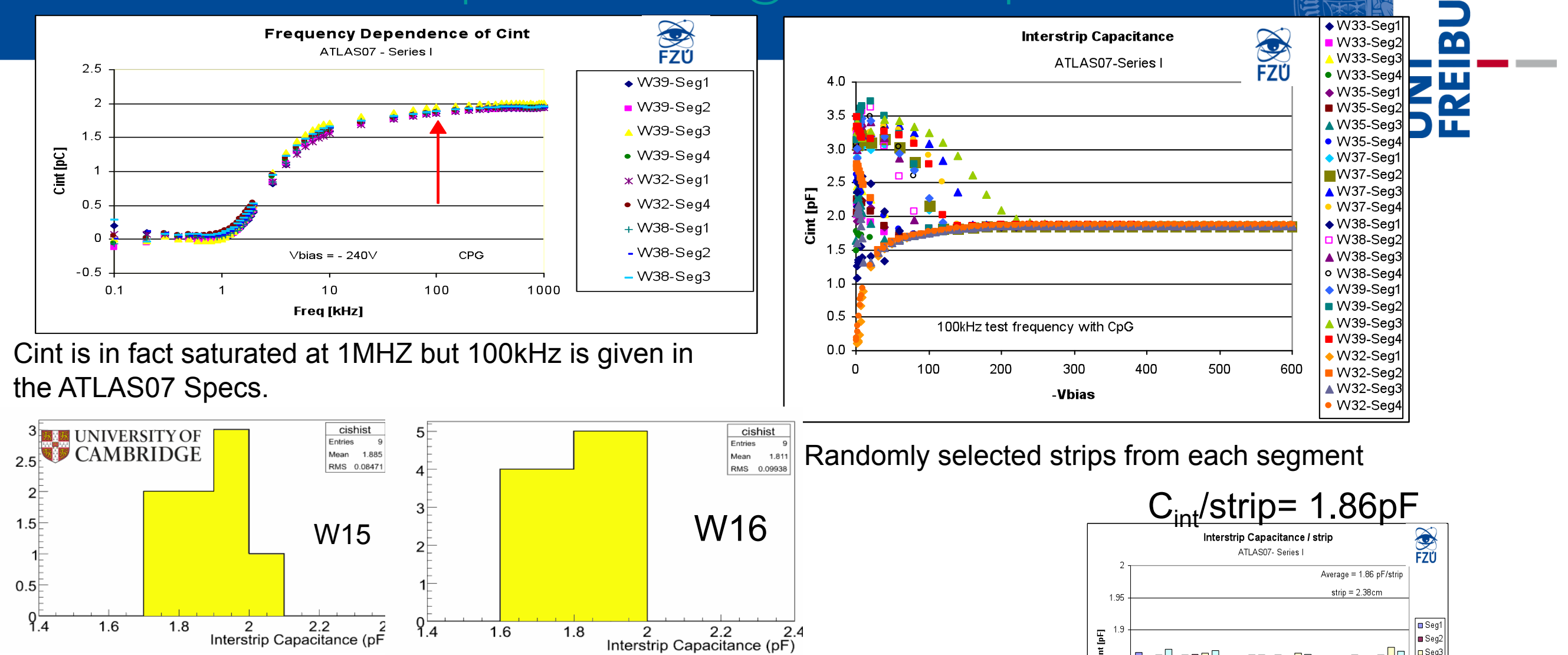

All tested sensors have Cint $\sim 0.75-0.80 \mathrm{pF} / \mathrm{cm}<1.1 \mathrm{pF} /$ Ala asurements taken on central strip with either neighbour grounded. Including next-to-neighbours results in 10-15\% higher readings.

Randomly selected strips from each segment

Cint contributes dominantely to the input capacitance of FE electronics and determines noise level and therefore should be as low as possible.

Cbulk $=3.25 \mathrm{nF}$ at FDV for full area sensor but one strip capacitance is equal to $\sim 0.6 \mathrm{pF}$ only.

It is 3 times smaller value than measured Cint $=1.86 \mathrm{pF} /$ strip $_{\text {Lviik }}$ - ATLAS Tracker Upprade 


\section{Backup}

\section{Leakage Current (@500/800V) with protons}

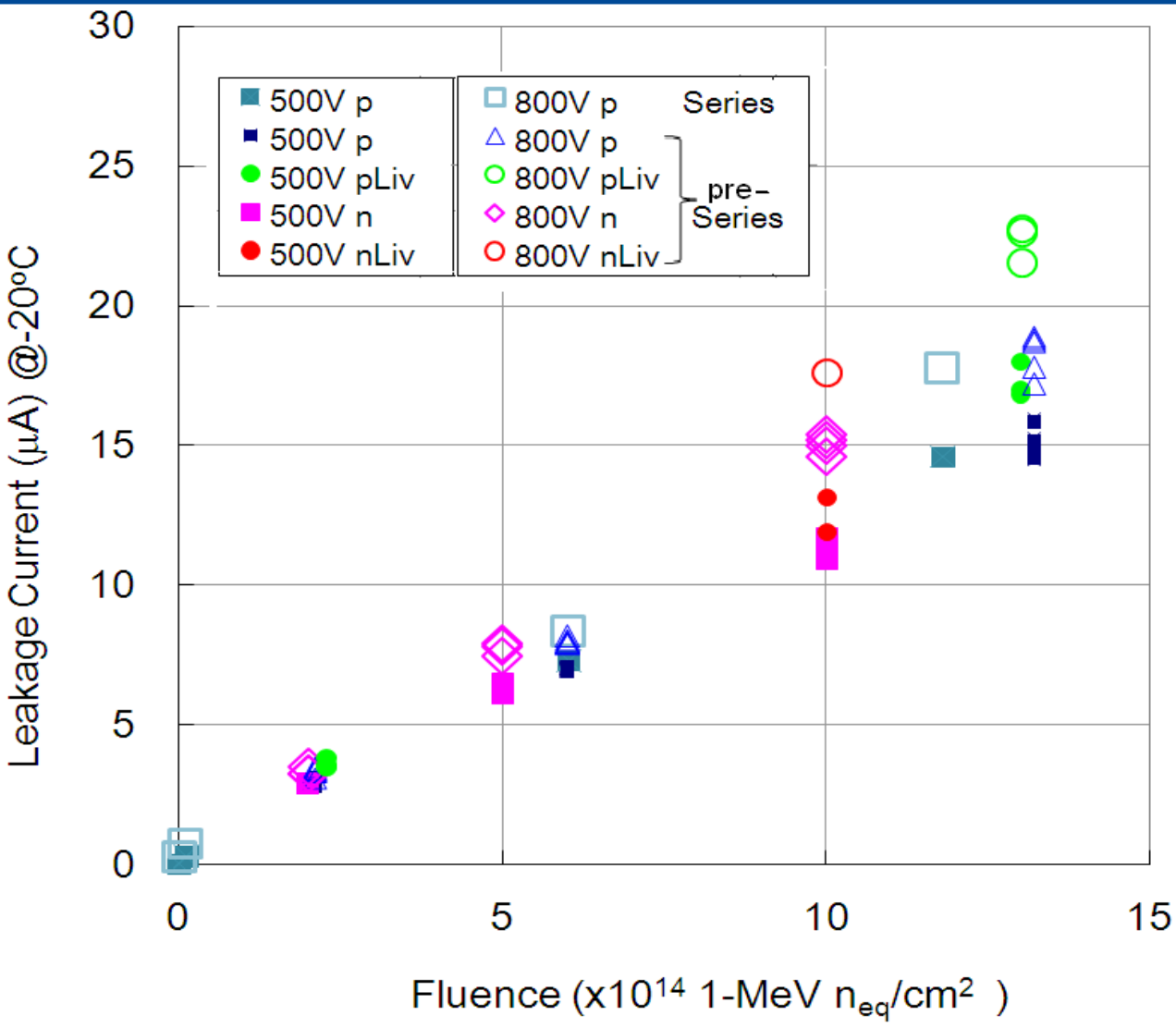

Current $/ \mathrm{V}=\alpha \cdot \phi$

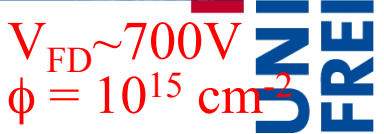

$>$ The damage constant (slope)

$\sim$ consistent with n-bulk damage constant $\left(\alpha \sim 4 \times 10^{-17} \mathrm{~A} / \mathrm{cm}\right)$

$>$ The leakage current of non-irradiated p-bulk sensors is at the similar level to HPK n-bulk sensors

\section{Agreement between sites (Tsukuba/} KEK and Liverpool)

\section{proton/neutron damages contribute} similarly to leakage current increase

Fluence $\left(x 10^{14} 1-\mathrm{MeV} \mathrm{n}_{\mathrm{eq}} / \mathrm{cm}^{2}\right)$

K. Hara et al., "Testing of bulk radiation damage of n-in-p silicon sensor for very high radiation environment" , $7^{\text {th }}$ International "Hiroshima" Symposium on the Development and Applications of Semiconductor Tracking Detectors. 